\title{
Philosophy of Conceptual Network
}

\author{
Bernard Korzeniewski \\ Faculty of Biochemistry, Biophysics and Biotechnology, Jagiellonian University, Kraków, Poland \\ Email: bernard.korzeniewski@gmail.com
}

Received 17 August 2014; revised 15 September 2014; accepted 6 October 2014

Copyright (C) 2014 by author and Scientific Research Publishing Inc.

This work is licensed under the Creative Commons Attribution International License (CC BY).

http://creativecommons.org/licenses/by/4.0/

(c) (i) Open Access

\section{Preface}

The article formulates the philosophy of a conceptual network and defines a concept as a unit of meaning. According to the proposed idea, the substance of our mind (subjective psyche) is constituted by a conceptual network which is composed of continuous concepts which have meanings through connotation. The conceptual network is an epiphenomenon of the neural network that is based on a dynamic structure of a complex of neurons interconnected in a functional way. Language is a secondary phenomenon in relation to the conceptual network: the words of language correspond to the best-distinguished concepts, and their meaning is determined by their conceptual "lining". The conceptual networks of logic, mathematics and, especially, philosophy are not perfectly determined and specified. The "Absolute Truth" not only does not exist, but in fact it does not have any sense. (Self-)consciousness emerged as a result of recurrent self-reference of the neural/conceptual network.

\section{Keywords}

\section{Concept Network, Meaning by Connotation, Language, Epistemology, Absolute Truth}

\section{The Absolute Truth}

The "absolute" truth is silence. If we decide to say anything more, we do so only to eventually return to the above statement, but keeping the memory of our journey in our consciousness. We do it in order to demonstrate that what is not the absolute truth is all we know.

\section{The Conceptual Network}

We will start from a concept, but we will not define it now-this entire work will constitute its definition; the concept of a concept will be involved in all sorts of relations with other concepts. Let us begin with the colloquial, intuitive meaning of the word. Here, we will name only a few of its properties which seem to be essential in this part of our argument. 
First of all, a concept is a continuous, unquantized entity (in contrast, for example, to the names of language). This means that a concept (being a certain unit of meaning or sense) does not have sharp boundaries with other concepts (meanings), but it manifests its presence and individuality through greater intensity in the "field of meanings" (the semantic field). We could compare it to a hill in a landscape: although we can clearly see the hill, as if extracting it from the landscape, we cannot describe precisely where it ends and a valley begins, nor can we draw a sharp boundary between hills. Thus, the "existence" of hills is not inconsistent with the continuity of the terrain, the smooth transition from one form into another. Of course, concepts are not in "real", three-dimensional space, but in the space of meanings, where heights are matched by the intensity of the semantic field, and it is pointless to define its dimensionality (the number of dimensions of this space may be assumed to be potentially infinite).

This does not mean that a field of meaning exists in an objective or absolute way, but only that it is a convenient model which allows us to describe interesting properties of concepts (which will later turn out to be nothing absolute either). Continuity between concepts exists both "horizontally" and "vertically", that is, both between the concepts of the same degree of generality, and between the concepts of different degrees of generality. The latter case of continuity will be called the non-hierarchicality of a concept. Thus, concepts are the most specific and tangible meanings as well as the most general and abstract ones. There are no sharply separated concepts, or levels of generality, or any other categories - they all flow seamlessly from one to another. This is a necessary and sufficient condition for any relation between them. If we have a continuous field of concepts, then its axes are both a diversity of concepts at the same level of complexity, and a concept's degree of complexity, its hierarchicality. Here, we should emphasize again that the terms "level of complexity" and "hierarchicality" have no practical sense except for drawing certain continuous axes which differentiate concepts in the field of meanings. At the level of the conceptual network, there are no simple, basic concepts, nor are there any complex, derivative concepts. All concepts are characterized by the same degree of simplicity (or complexity). The concepts are differentiated by their specification, which is a quantitative property identical with the intensity of the semantic field. But the meanings of concepts depend solely on their mutual relations. A very simplified example of the conceptual continuity—both "horizontal" and "vertical"—is provided by the following diagram:

- a red thing—a pink thing—a white thing.

- a red flower-a pink flower-a white flower.

- a red rose-a pink rose-a white rose.

In this scheme, which is constructed necessarily within the syntax of language, the dashes, of course, meanin accordance with what has been said above-the seamless transition between concepts (places of relatively higher intensity in the continuous field of meaning).

So far we have explicitly emphasized two properties of a concept: its continuity and non-hierarchicality. As we have already said, this entire work will further clarify the implicit meaning of the concept.

The concepts in the semantic field, which has some special properties, constitute a conceptual network. As in the case of the concept itself, we will not define the conceptual network. For the time being we will only say that the conceptual network is a set of concepts bound by specific semantic relations, which will be characterized below. We will not argue that "everything that exists is a conceptual network". It is simply a convenient model which allows us to describe well all the phenomena accessible to us and to define correctly relations between them. Apart from this function, we do not assign to it any existence which would be more or less real than the existence we assign to other phenomena. This approach is obviously derived from natural sciences. Undoubtedly, a model can be better or worse, that is, it can describe its object more or less correctly. A good model should describe a possibly wide spectrum of phenomena and should provide deep insight into the relations between them. It should also derive macroscopic properties of the whole system from microscopic properties of its constituents. Any model can be replaced by a better model, and for this reason it cannot aspire to any finality or absoluteness. At best, it is always only a sufficiently good temporary approximation. This differentiates the philosophical conception presented here from the majority of philosophical theories which attribute absolute validity to some assertions.

If the idea of a conceptual network seems somewhat unclear now, it should get clearer as we go deeper into this work. Various concepts appearing in it (including the concept of a concept) will gain more meaning gradually through reference to other concepts, both new ones, developed in this work, and those commonly used in the spheres of science, philosophy, and everyday life. Thus, only the whole conception presented here will constitute an adequate definition of the terms used in it. But returning to the conceptual network, we will name (as 
in the case of a concept) several of its characteristic properties here. The first two come directly from the properties of a concept. In particular, the properties of continuity and non-hierarchicality mean that it is impossible to draw a sharp boundary between a concept and a conceptual network. Continuity and the "equivalence" of concepts within a conceptual network is its fundamental property. The continuity of a conceptual network de facto implies the flattening of the hierarchy of generality to one level, if only we assume (intuitively obvious) categorial unity of its constituents (concepts). Thus, the concepts corresponding to such different "beings" as a stone, an angel, justice, and joy, belong to the same category due to the very fact that they are all concepts. Besides, the diversity of concepts goes far beyond their location on the axis: "less general-more general". Nevertheless, this diversity completely disappears at the conceptual network level. The only determinant of a concept in this network is its corresponding intensity in the field of meaning and its location in relation to other concepts (these properties are in fact interrelated). In short, the concepts only have meaning due to their connotations, in reference to other concepts. This is a very elementary and fundamental property. It means that the meaning of concepts is not based on their, however understood, direct correspondence to certain "beings" outside the conceptual network, that is, on their denotation (this is a secondary phenomenon), but on their relations to other concepts. Concepts emerge through "polarization" on "semantic axes" in relation to other concepts "surrounding" them. Examples of simple semantic axes are: warm—cold, pleasant—unpleasant, large—small, good—bad. We should note here again that the semantic axes, like concepts, are continuous: both "along" an axis and "between axes", when we separate one axis from another. Besides, the kind of polarization (the opposite ends of an axis) is also defined by concepts. Here we see connotationality (concepts are defined by conceptual axes, and the axes-by the concepts), but also reflexivity, which we will address in a moment.

Is there really a continuous transition between the ends of the axis of meanings? For example, how is it with a pair of elementary particles: electron-positron? Both have the same mass and other properties, including the amount of electrical charge, and they differ only in the sign of the charge (an electron has a negative charge, and a positron - a positive one). Does the continuity of semantic axis not mean that there should be a lot of particles with transitional charges, including a particle with the properties of an electron and positron with a zero charge? No, because we should not mix semantic axes with real-existing objects which are only described by very few possible combinations of different axes of meanings. All right, but isn't the semantic opposition positive-negative rather discrete than continuous? No, because in order to distinguish the positive and the negative, we must arbitrarily designate a zero point at an axis describing a particular property, and what will fall on one side of this point, define as positive, and what will fall on the other side—as negative, but the axis will still remain continuous. But doesn't Nature herself sometimes set a zero point as in some way absolutely singled out? After all, a particle with a positive charge will be repelled by another positively charged particle, and a particle with a negative charge will be attracted to it, whereas a particle with no charge will remain motionless. However, if we replace the positive with the negative, the situation will remain exactly the same. Furthermore, the distinction "positive-negative" is determined by the direction of a particle's motion, and there is a continuous transition between all directions in space (the fact that repulsive particles have motion vectors that differ by 180 degrees is a feature of physical space, and not of semantic space; in the latter all possible combinations of vector directions are possible). Finally, there is no sharp boundary between motion and stillness-the speed of this motion can be freely close to zero. Thus, the discrete differentiation "positive-negative" is a secondary feature of our mind which uses discrete names of language, and not a property of the conceptual network and its semantic axes. However, as we will show below, "quantized" language is a derivative part of the conceptual network, and the absolute specification, the discreteness of its names is an illusion.

We have stated above that concepts have meaning through relations to other concepts in the continuous conceptual network. In turn, the intensity of the field of meaning, the clarity of a concept, is equivalent to its specificity. The concept appears to be more intuitively clear and obvious, the deeper it is grounded in the conceptual network, the denser its "semantic environment" is, and the more concepts it is related to. Thus, neither the meaning, nor "intensity" of a concept is its inherent feature; these features are determined by the surrounding concepts in the conceptual network. These, in turn, also do not mean anything by themselves, of course; that is precisely the essence of connotationality. So concepts appear to us more clearly, the denser the semantic network that surrounds them, and what they mean is determined by the kind of concepts that surround them. If we identify "existence" with "being a concept", then something exists the more, the deeper it is in the conceptual network, the more it is related to other concepts.

Two further properties of the conceptual network are reflexivity and universality. It is mainly because of these 
properties that we have taken the system of concepts as the subject of our discussion. They determine the usefulness of the conception of the conceptual network in philosophy. Universality means that the conceptual network in the form specified in this work allows us to grasp all the phenomena accessible to us. We can assign to a concept any kind of being in any form of existence, from the most real and tangible to the most general, mythical, or virtual entities, to the world of ideas, values, and feelings. Anything you can think of, imagine, or "grasp" in any other way, "is" a concept (or has a corresponding concept, which is an equivalent statement in this conception). Because whatever we perceive, it must mean something to us. So the property of universality is very important. It is not present in the conception of matter, spirit, monads, consciousness, will, the four elements, or in Wittgenstein's philosophy of language (unless, of course, we brutally substitute the name "everything" with any of the names mentioned above). For clarity, I will add that I do not embrace idealism, so I do not claim that there is no external world "out there" beyond the conceptual network; I claim only that the conceptual network is all that is directly accessible to us. The second, at least equally important property of a concept is its reflexivity. This property consists of a simple claim that a concept is also a concept, like many others. It is difficult to overstate the importance of this property.

So far our discussions seem to have led, more or less directly, to a somewhat strange conclusion that "everything is a concept". Because if in philosophy we give an absolute meaning to a being, such as matter, then we can refer all the phenomena accessible to us to this being, recognizing it as the essence of the world. However, this approach is unaesthetic and self-contradictory (more on this later). Materialism is simply not a reflexive system. The conception of a conceptual network is different. The concept of a concept, which is the basis of this system, also means only through relations with other concepts; it "exists" only in reference to them. Thus, it cannot be absolutely legitimized to constitute a foundation of a philosophical system, which it is also a part of, within which it operates. It cannot validate itself. And this, although it may seem to be a fault of the system, is its great virtue, in our opinion. Because the system shows its own lack of legitimacy, it negates itself in an absolute sense. Combined with the property of universality (the fact of embracing all possible phenomena), it means the delegitimization of everything in the absolute sense, the nonsense of making valid claims regardless of reference, the emptiness of the concept of the "absolute truth". In other words, due to the conceptual (and therefore relational, or relative) nature of our thinking, it is impossible to construct within our consciousness a system which would be meaningful in an absolute, autonomous sense, that is, irrespectively of its semantic context. This also applies to the conception presented here. We believe that its main virtue is the fact that it is possible to recognize within it this state of affairs.

At this point, we have made our first return to the assertion at the beginning of this work; for the first time we have closed the circle leading to a denial of absolute truth, to the nonsense of absolutely "objective" claims. We will make more such returns, from various reference points, from different places within the system. When we assume the "existence" of a concept, we proceed as we would do in mathematics or physics, where for the purpose of proof, in some cases, the "existence" of imaginary numbers is "assumed", but only to reject it and return to the world of real numbers after completing the proof. The debt to the principles of legitimacy (here we are talking about physics, because in mathematics everything is permitted) was paid off, and we have pure profit from this operation in the form of the proof. This way, of course, we deny-following in the footsteps of early Wittgenstein (Wittgenstein, 1961)—absolute validity of these discussions. But, first, the bare statement "the absolute truth doesn't exist" means something different than the same statement involved in the whole web of interpretations and specifications; and second, if the absolute truth is nonsense, then relative truths are all we have. The aim of this work is to evoke in the reader a feeling of "truthfulness" of the presented conception, to find a way with this "conceptual vision" to the system of concepts formed in his or her mind. Conscious abandonment of all forms of the Absolute is not easy. In particular, we deny the existence of any absolute meanings. However, by abandoning the Absolute we can, paradoxically, say more about the world, than when we insist on the Absolute's existence.

Connotationality of concepts can be expressed in various ways. We could remove one node (concept) from a conceptual network, and patch the resulting hole up by "pulling” neighbouring concepts (relativized to it) into its place. An example of this operation in the sphere of language is provided by Russell's method of description (Russell, 1905) or Hume's analysis of the concept of “cause” (Hume, 2007). Then, it turns out that the analysed concept is empty, that it has no designatum. And most importantly, such analysis can be carried out for any concept. It is a kind of equivalent of defining names in a language by other names (the whole of the next section will be devoted to the relation of language to the conceptual network). Proceeding in this way consistently, we 
could completely dismantle the entire conceptual network. It is a reverse process to the emergence of the conceptual network, which we will deal with later in our discussion. Another example of connotationality is transferring concepts to another semantic context. If a given concept emerged in a certain conceptual environment (let us say, in everyday language), and then, due to some analogies in the "conceptual situation", we move it to a different environment, usually under the same name of a language, then basically we have a different concept. However, a shared name often leads to equating these concepts and creates illusory problems. Later in this work we will discuss a well-known, but in fact senseless question: whether mathematics is discovered or invented (Barrow, 1992). Connotationality also determines the property of reflexivity; in practice all the properties of the conceptual network can be, more or less directly, derived from other properties, which is also-at another level-an expression of the connotationality of concepts (the property of connotationality is inextricably intertwined with reflexivity). The denser the conceptual network is in a given "region", and the richer the relations between concepts are, the easier it is to embed or create new concepts (it is positive feedback). In addition, concepts are considered to be more essential, "real", specified, indisputable, and obvious, the deeper they are involved in relations with other concepts. Therefore, e.g., any religion is something more than a simple assertion of God, Purpose, or Hope. Each religion has its own mythological surroundings which strengthen the concept of God and expand His connection to the material world. Within the system of concepts, God is more "real", the more things that connect him to "reality".

In order to clearly see the connotationality of concepts, it is sufficient to make a small psychological observation. You should select a concept (the more abstract it is, the easier it will be to achieve this effect) and focus strongly on it and on its meaning. After a while, the essence of this meaning will begin to dissipate in our consciousness, it will be ceded to neighbouring concepts in our conceptual network, which we are not currently focused on. In the end, we will be left with an empty linguistic name, and for the time being, we will not be able to assign any meaning to it. This phenomenon is analogous to the logical analysis of the names of language.

Limitations in the study of the conceptual network are to some extent analogous to Heisenberg's uncertainty principle (Hawking, 1988; Penrose, 1990). The more "analytical power" we apply, i.e., the more concepts we substitute with definitions constructed out of other concepts, the less the other concepts will be specified (and therefore "semantically localized"). As we eliminate more and more concepts, the number of connotational relations of existing concepts decreases. Therefore, these are no longer the same concepts. Each consistently performed analysis of the conceptual network leads to conceptual emptiness. Analytical methods, including Russell's method of description (Russell, 1905) and Wittgenstein's programme of the "clarification of sentences" (Wittgenstein, 1961), not to mention the logical positivists, were developed with the assumption of semantic absolutism (to be discussed below). Thus, they did not take into account the fact that the meanings of concepts (and thus their identities) are not autonomous, but depend on surrounding concepts. On a small scale, conceptual analysis is good for eliminating "conceptual excess" and "locally meaningless" concepts (due to reflexivity, all concepts are globally meaningless), primarily in philosophy, but when used consistently, it leads to "desemantization" of the entire conceptual network.

If we treat the conceptual network as a kind of material, then the whole body constructed with this material we will call the crystal of concepts. Obviously, its structure is not homogeneous. The conceptual network creates some partly separated regions within the crystal-conceptual maps (this name was taken from differential geometry, where maps cover a curved space, whereas conceptual maps cover all accessible phenomena). And again - separation of these maps is neither sharp, nor absolute, they continuously flow one into another. These maps are simply areas in the crystal of concepts characterized by a relatively higher density and better specification of concepts, closer interrelations within a map than between maps. If we make the analogy to a landscape again, then the conceptual maps are ranges of hills separated by valleys that are wider than individual hills (concepts), of course, with the continuity of the landscape preserved. Pursuing this analogy further, we find a certain hierarchy of maps' sizes, as the ranges of hills combine to form larger complexes, and these, in turn, form whole geographic regions. In both cases, however, the imposed hierarchy and the divisions made within it are completely arbitrary. They can facilitate description of a given structure (geographical or conceptual), but they should not be regarded as proof of the existence of some absolute categories. To limit this analogy, however, we should remember that while the structure of landscape is three-dimensional, it is pointless to define the dimensionality of the conceptual network.

As examples of conceptual maps, we can take the areas of everyday life, science, religion, and aesthetics. The map of science includes the maps of its disciplines, within natural and humanistic sciences. On each map, local 
concepts are involved in closer relations than with the concepts from other maps. Of course, many concepts are parts of many different maps. However, due to somewhat different semantic context, these are actually different concepts hiding under the same linguistic name. Paradigms in science are an example of another category of conceptual maps. They divide the "supermap" of science in a completely different way than the maps of its disciplines do (as when a landscape could be divided into different hills, and, quite independently, into forested and deforested areas). The structure of a conceptual map has some characteristic features: its centre is "denser", it includes more specific concepts, whereas its periphery is occupied by concepts less distinguished and worsedefined by neighbouring concepts. If a given edge of the map is neighbouring on another map (at this point, analogies to three-dimensional terrain can be very misleading, as this neighbouring can take place within a huge number of semantic dimensions), then a higher density region starts "over there" again. However, if a given edge is not neighbouring on any other map, then concepts gradually dissipate in a conceptual void. Philosophy per se and some areas of physics are examples of this phenomenon; this issue will be discussed in more detail later in this work. When maps have contact with one another, they change smoothly into another; in particular, in the crystal of concepts there can be no two maps which are not, directly or indirectly, in contact with each other. In the area of contact there is "agreeing" of maps (this term is also taken from differential geometry). Some concepts from different maps with a slightly different conceptual environment within them are "equated" with one another, and that is why it is possible to translate fragments of maps lying outside the contact area into another. But because this "equation" is necessarily an approximation (equated concepts are not fully identical), only the fragments of maps close to the point of contact can be translated moderately well. The further away from the point of contact, the less accurate the "translation" is, and eventually it loses its legitimacy. The effects of this "equation" get worse the more distant the maps are from which the concepts were equated. The above-mentioned question of whether mathematics is invented or discovered may serve as an example. The equation of concepts from everyday life and mathematics leads to meaningless situations, e.g., when we move the whole conceptual environment of a given concept from everyday life to the conceptual map of mathematics. This issue is developed later in the work.

To illustrate the phenomena of construction of conceptual maps, their mutual contact, and dissipation of their peripheries in the semantic void, we will use an example from the field of exact/natural sciences. Let us consider physics, chemistry, and biology. All these sciences are relatively exact and well-defined at their centres (of course, there are clear subdivisions within them, but we will not deal with them now; the argument in relation to them is analogous to that at a higher level of hierarchy). This means that within their range they are governed by a set of well-defined laws specific to each of them. The density of the conceptual network, and therefore specification of the concepts, is high at their centres. For example, biology uses the concept of species freely, although the concept has not been defined precisely yet. But it is so widely used and so useful in practice that its certain vagueness is neglected. This phenomenon is a general feature of the entire crystal of concepts- there are no "perfectly" defined concepts, the degree of their definition depends on the number of relations with other concepts. However, in regions where one science changes into another, the concepts are less defined. Physics is not able to describe atoms accurately, except for a hydrogen atom, and therefore it provides only approximate foundations for chemistry. Besides, and more importantly, quantum physics is based on probabilistic wave functions, whereas classical chemistry is deterministic at the microscopic level (of course, similar problems exist within physics itself, but we neglect them now for the sake of simplicity). Similarly, chemistry, although it describes (through biochemistry) reactions taking place in a living organism, is not able to explain life-life cannot be derived from chemistry. A line of contact between biology and psychology is an even better example. For the phenomenon of mind cannot be fully explained by the principles of brain functioning, despite great progress in neurophysiology. In general, the problem of reductionism is related to inaccurate agreeing of maps on the line of contact. This becomes evident when we realize that each of these maps has developed in relative isolation, and has worked out its own concepts, laws, and systems of meanings. These concepts can be compared to analogous concepts on other maps, but this reference is only approximate due to differences in conceptual environments.

It is sufficient to compare the meaning of the concept of "time" in physics, chemistry, biology, and psychology. For a physicist-dynamicist, time is primarily an integral part of curved space-time, its component as symmetrical and "reversible" as spatial dimensions. For a physicist-thermodynamicist, there is a clear arrow of time as an indication of irreversible processes and the growth of entropy. For a chemist, time is associated with a parameter describing the kinetics of chemical reactions (these are periods measured in seconds and minutes). Biology uses much more diverse timescales, different for evolution, ontogenetic development, and physiological, 
biochemical, or biophysical processes. Finally, the subjective time of psychology is impossible to compare with any of the others. Even for such close fields as equilibrium and non-equilibrium chemistry, time has a completely different meaning.

Another example is the phenomenon of biological evolution, which has no equivalent in physics and chemistry. That which we usually call the evolution of the Universe, of planet Earth, or, more rarely, the evolution of a chemical system over time, is simply a sequence of transformations of consecutive states, and these transformations are-at least at the macroscopic level—determined and do not increase the complexity of the system. In addition, normally, physical and chemical systems do not retain the memory of their previous states. In contrast, biological evolution is a process which collects information about past "experiences" of the system, and often leads to the increase of complexity of the system (whether it is a biological cell, tissue, or a whole organism, population, or biosphere). Thus, the concept of evolution may be only roughly agreed on between physics and biology, in the sense of a system change over time, but in fact these are two different processes and two different concepts hiding under the same linguistic name. The second reason is that biological evolution cannot only be explained within a conceptual map of physics - we need a conceptual apparatus developed by the theory of evolution. Cultural evolution is still another thing. Apart from significant differences in the subject of evolution (in cultural evolution there is a very important psychological factor), there are other ways of accumulating experiences and gathering information (it is difficult to overestimate the role of language, and especially the oral and written communication of information in cultural evolution). And finally, whereas biological evolution is opportunistic (it works on the "trial and error" principle), in cultural evolution a large part of development is played by the active mental factor.

In addition, the concept of "cause" means something slightly different in various branches of physics, chemistry, and biology. In astronomy, the "cause" is a simple and in principle fully determined gravitational force. For example, we can calculate with practically any level of accuracy the motion of two interacting bodies in space (if the gravitational pull of other bodies is negligibly small). In quantum mechanics, the cause boils down to the probability of an incident described by the spatial distribution of wave function. In chemistry, the cause is the law of large numbers referring to a huge collection of molecules. In physiology, the cause is a large and often unknown number of mutually reinforcing or suppressive stimuli, which as a result give an effect described only probabilistically. Finally, in evolutionism the cause is basically reduced to a certain set of general laws governing the process of evolution (e.g., natural selection) and to random events.

Assigning various concepts to the same linguistic names also leads to conflicts between science, religion, and everyday life. The often proclaimed unity of physics or exact/natural sciences in general is therefore fiction. It has been said that science is a sequence of successive approximations ending in infinity. However, taking into account the connotational nature of the conceptual network, we should rather say that it is a sequence of consecutive clarifications. The question of whether the lack of legitimacy of reductionism is a temporary phenomenon resulting from our ignorance, or an ontological one-does not matter at the moment. One can only conclude that a possible "agreement" of adequate concepts will never be perfect and it will be the worse the further away the maps are from where the concepts we are trying to agree are taken. Thus, if we ever manage to unify or "fuse" the maps of physics and chemistry (or the map of quantum mechanics with that of the general theory of relativity) with "sufficiently good approximation", then the possible indirect (through chemistry and biology) fusion of the maps of physics and psychology will be much less satisfactory. It is simply as the agreement of two maps reduces the ability of the resulting map to merge with any other map-it will be more difficult to agree with it than any of the starting maps individually. Agreement of conceptual maps improves specifications of concepts. The semantic environment of a concept in the agreed-on map is richer than in the starting maps, and its attributes are better specified. As a result, a concept becomes less equipotential, has fewer possibilities of further clarifications. This increases the differences between this concept and the corresponding concepts in the maps that have not yet been agreed on or have been agreed on separately. In the end, the difference becomes so large that we have de facto different concepts despite a single corresponding linguistic name. And it is difficult to agree on maps which do not have any shared concepts.

As a comparison, we can take a straight-line tangent to a circle at point $\mathrm{X}$. At a very close distance to this point, the straight line is a good approximation of the circle, but as the distance increases more and more, this approximation becomes worse and worse, and eventually loses any meaning at all. In this analogy, the circle symbolizes a reality, and the straight line-a conceptual map that describes this reality. It is possible to agree with good approximation only the adjacent maps. The effort put into each subsequent unification will increase 
exponentially, and the resulting unified map will be much less prone to agreement with other maps than the starting maps unified within it. "The end product" of this process would be a large, well-unified map unable to further agreement with other maps. It is for this reason that the Grand Unification of Science is a utopia. We can observe the growing difficulty of unification in physics. While Newton's unification of falling and orbital motion in his theory of gravity, or the unification of magnetism and electricity in Maxwell's equations, was relatively easy, we required the genius of Einstein to equate matter with energy, or the curvature of space-time with gravity; and the Grand Unification of all four elemental forces, despite years of attempts and increasingly breakneck mathematical constructions, still remains beyond our reach. The same applies to a potential agreement of conceptual maps of quantum mechanics and the general theory of relativity.

Of course, both the history of the development of various conceptual maps and the story of their possible unifications, has a fundamental influence on the structure of the crystal of concepts and possibilities of further unifications. The division of natural sciences into separate disciplines corresponds more to the historical emergence of conceptual maps, than to "real" diversity of natural phenomena. For example, chemistry is no less "physical" than crystallography or solid state physics, and the differences between them are certainly smaller than the differences separating these two disciplines from the general theory of relativity. Problems of reductionism consist of the difficulty in agreeing maps which originally developed relatively independently, with separate "centres of crystallization" of meanings in the conceptual network. Each of these maps drew on everyday language, but within the maps, the same names gained slightly different meanings; a new vocabulary emerged, specific for individual maps. When it came to agreeing the maps, they turned out to differ in some dimensions of the semantic space, so that it was possible to carry out only a rough correspondence between concepts in adjacent maps. This fact is a fundamental obstacle for agreeing and unifying maps. Achieving our crystal of concepts in its current form, we have lost billions of other possibilities and we will never know how they may look. Further specification of concepts is an irreversible and cumulative process. We cannot even reject all our knowledge and start again from scratch, as we cannot reject our crystal of concepts, shaped by this knowledge. We are not able to look at it from the outside. Along with the crystal of concepts, our existence would have changed, and therefore our ontology as well.

As we have come to theoretical physics, let us use it as a good example of dissipation of concepts in the semantic void on the edge of the crystal of concepts. Such concepts as "matter", "causality", and "event", which are well known to us from everyday language, lose their meaning, e.g., in quantum mechanics, they "slip" through the fingers, break up their connotational bond with other concepts, until finally we see emptiness instead of clear and obvious concepts. Matter is "melting" in field intensities, wave functions, probabilities, and equivalencies with energy, so that finally we cannot say exactly what we really mean when we say "matter". When we have subtracted all of its attributes, such as weight, extension, colour, and locality in space, we have completely "undefined" the concept of matter, broken it down to its most basic constituents-the concepts surrounding it. There is a similar situation with the concepts of "causality", "event", and many others. This is simply an area where our conceptual map is in contact with the semantic void-this is the edge of the crystal of concepts. On a larger scale, this process occurs in philosophy, where each analytical treatment of a statement demonstrates its deceptiveness, nonsense, and lack of legitimacy; where it is so easy to use the method of logical analysis in order to find contradictions and meaningless sentences as understood by early Wittgenstein and the logical positivists. How can we better specify semantically this dissipating edge of the crystal of concepts? Only by semantic "thickening" of these regions of the crystal of concepts which lie slightly deeper. We will return to this topic while analysing the influence of physics (or, more broadly speaking, all the exact and natural science) on philosophy.

We say that theoretical physics lies on the edge of the crystal of concepts, i.e., it borders with the conceptual void, because, like philosophy, it speaks, in a sense, what "everything" is. When it explains the phenomena it studies, it cannot appeal-as other disciplines do—to anything outside itself. The issue of reductionism is the most glaring example of this predicament. Living organisms can be in a way "explained" (or at least we can try to explain them) by referring to chemistry, and chemical phenomena can be quite well described in the language of physics. But physics, lying at the bottom of this reductionist progression, has to rely only on itself. Physics tells us about the essence of the world, at least of the material world at the lowest level of complexity known to us (which does not mean that it is simple-physics is the science which most defies our common sense!). Physics describes such fundamental properties of the world as the local structure of all its "objects", including human beings and the geometry of the whole. Physics explains, at least in an outline, the phenomenon of life (thermo- 
dynamics). It explains how the world emerged and predicts how it will end. Thus, it is very close to philosophy. The latter borders with the conceptual void in an obvious way, it constitutes something like a "conceptual buffer" between itself and well-specified conceptual maps within the crystal of concepts. The intensity of the semantic field cannot suddenly fall from high values to zero. Philosophy ensures a gradual transition. Therefore, it is hardly surprising that the concepts within it are poorly defined, that there are many easily detectable contradictions, and there is a huge freedom to manipulate meanings. Philosophy could not exist without its "foundation" in the form of science, religion, and everyday life. It cannot develop without them, especially without science.

How is it possible that we can meaningfully use concepts at all, if a consistent analysis of each of them leads to the semantic void and each concept is defined by other concepts? These other concepts, in turn, are defined by yet other concepts, until finally the circle is closed-and we come to the conclusion that all concepts define all other concepts. But this is the essence of the connotationality of meaning. Fortunately, in practice, we do not carry out our analysis consistently to the very end. We "leave alone" the concepts which define the concept interesting to us, we intuitively take them at face value, and provisionally assign to them a degree of absoluteness.

Let us analyse this using an example. A benzene ring consists of six carbon atoms. Of course, chemistry and physics exactly define a carbon atom. It consists of a certain number of protons, neutrons, and electrons, and it has chemical properties resulting from its construction. Protons and neutrons, in turn, are composed of quarks. The probability of finding a given electron in a given point of space is determined by wave function. And so on. However, when we say that a benzene ring is composed of six carbon atoms, we often do not remember about these facts at all. It is enough that the name "carbon" is intuitively familiar to us and we have an impression that we know what carbon is (and more specifically, what a carbon atom is). To someone who does not know the quoted facts of chemistry and physics, the name "carbon atom" is more or less semantically empty. But it is sufficient that we are accustomed to a given name, even without knowing too well what it designates, and we can use it quite well with a subjective sense of understanding. In fact, we could even use a name that is semantically completely empty. If we decide that a benzene ring is a six SOMETHING arranged in a ring, and butane (or rather its carbon skeleton) is a four SOMETHING arranged (approximately) linearly, then we somehow understand these definitions, and they are not a complete nonsense to us. These definitions mean something to us in relation to another (and, of course, in relation to many other concepts or definitions). They differ between themselves by their location on two semantic axes. The first is the axis of number and the second is the axis of spatial distribution. These axes are not absolute; they contain sub-axes and consist of concepts, although this is not important at the moment. The above analysis is yet another example of meaning through connotation. Thus, if we can create meaningful definitions containing empty names (or, in other words, extremely vague names), then it does not surprise us anymore that we can freely use the name "carbon atom" or "electron", not really knowing what it is (for that matter, no one really knows what an electron is). A very poorly specified concept (or a name) may become a part of meaningful sentences by entering into various configurations with other concepts. Thus, the fact that each systematic analysis of a given concept leads to a semantic void is not inconsistent with the subjective feeling of its understanding in a "semantically dense" area of the conceptual network. It is impossible to carry out a consistent semantic analysis of the whole crystal of concepts, if only for the reason that the analytical apparatus is also a complex conceptual system and its desemantization in the process of analysis would prevent the continuation of this process. For the above reasons, despite the connotationality of the conceptual network and the possibility (at least in principle) of complete decomposition of each concept into others, we do not see the conceptual network as a semantically empty construction—on the contrary, its meanings seem clear and unambiguous to us.

One might ask why instead of saying "everything is a concept" (this statement is important only in a certain context), we do not simply say "everything is everything", if we claim that the conceptual network covers all phenomena accessible to us. Is not the system presented here simply a tautology? The answer to this objection provides, paradoxically, important information about the discussed conception. The thinking presented in this objection only reveals non-legitimacy of logic operating on the linguistic names (we will deal with this more extensively in a subsequent dedicated section). To us, of crucial importance is the difference between the conceptual environment of the names "everything" and "concept". The former is so general that it is impossible to say anything with it. In contrast, the usefulness of the name "concept" in an "operational" sense has already been demonstrated. The "concept" is simply associated with a greater number of other concepts relativized to it, than "everything". Undoubtedly, the statement "everything is everything" is closer to "truth". However, because as a tautology it is practically empty semantically, by expressing it we return to the statement from the beginning 
of this work that the absolute truth is silence. This way, the objection confirms, after deeper analysis, the theses presented in this work.

\section{The Conceptual Network and Language}

In order to specify the conceptual network better, we will take language as a point of reference. When we want to describe a completely new phenomenon, it is good-apart from naming its characteristic features-to compare it with a well-known phenomenon. In which case, the revealed similarities and differences will help us place the new phenomenon among those phenomena which are intuitively obvious and well-grounded in the existing conceptual network. This is consistent with the idea of the connotationality of meanings. The first phenomenon, in relation to which we will relativize the crystal of concepts, is language, basically as viewed in Wittgenstein's Tractatus Logico-Philosophicus (Wittgenstein, 1961), but also in everyday sense.

First of all, we will compare the ranges of the crystal of concepts and language, assuming some-unspecified so far-correspondence between them. It seems that we can assume that these ranges are roughly the same, at least potentially. This means that if we take a sufficiently large fragment of the conceptual network, it will have some corresponding language element, and the other way round. Thus, even if the ranges of the language and the crystal of concepts do not coincide exactly, that is, not every element of one of them corresponds to an element of the second, then by using a sufficiently large "scale of the map", and sufficiently rough "grain of the picture", we can get an approximate "congruence" of the language and the crystal of concepts. Both these phenomena refer to "reality" and to each other through denotation, correspondence of their elements (i.e., a concept is matched by a name, and the name is matched by a real "fact").

The first fundamental statement is that the denotation relation "the crystal of concepts-language" and "language- 'reality"' is secondary to the connotation relation within the conceptual network. This means that the denotation relation itself is also a part of the conceptual network and thus it is "created" by the concepts which have meanings through connotation. In order to clarify the above sentence we will address further relations "language- the crystal of concepts". The basic difference separating language from the conceptual network is its "quantization". This means that the words of language are separate, distinct units which differ among themselves by names. There are no continuous transitions between one name and another. Of course, we can create intermediary names, such as "whitepink" to describe a transitional colour between white and pink, but this does not change the quantization of language at all. This feature means the impossibility of connotation in the sphere of language; later we will show that defining names (their meanings) takes place in the conceptual realm, and not in the linguistic one. The quantization of language makes it clearly different from the continuous conceptual network.

How does denotation takes place between the words of language and concepts? This is the second very important feature: the names of language correspond to the areas of the conceptual network (concepts) which are most strongly marked, distinguished, and specified by their conceptual context. We simply assign names to the concepts which we subjectively perceive as sufficiently "individual" and distinct. Again, we can appeal to the analogy of hills in the landscape. Only the largest and most distinguished peaks have their own names and are perceived as separate hills. Small hills, not to mention individual boulders or pebbles, remain nameless, blending almost imperceptibly into the overall terrain. Indeed, a whole collection of the names of language or even a bigger part of it (a fragment of a literary, scientific, or philosophical work) may in fact correspond to one concept.

Something that constitutes the main idea of this work, its essence which is supposed to evoke a sense of understanding and the impression of truth, is also a concept. Its specification is provided by the whole body of discussions presented here. Undoubtedly, however, this concept reaches far beyond this text; it is being constructed on the already existing conceptual network and emanates with nameless meanings richer than those that are "literally" expressed in the text. We simply do not have names for concepts that are barely discernible, unspecified, and partly eluding our understanding. Here, we encounter an important consequence of the above statements: there are large areas of the conceptual network which have no equivalents in language (these are areas "between" well-specified concepts). All underspecified concepts, hints of meanings, and emerging ideas before they are clad in richer conceptual robes, elude language. All poetry serves to capture meanings which do not have simple names. And we receive it not through a particular configuration of words, but because the underlying concepts enter into new, as yet undiscovered semantic relations which we recognize as something that existed in us earlier, but was unnamed. 
When we express a thought, we often (in fact always, but to a various degree) feel that our statement is incomplete, as the linguistic name is necessarily poorer than the concept corresponding to it. Hence the saying that the uttered thought is a tomb of hundreds of expressions of this thought that have never seen the light of day. This example shows that we can assign to one concept a number of more or less fitting structures of language. Linguistic cognition, which is very important and stimulating for the development of the conceptual network, consists of assigning names (single or multi-word) to new concepts. This act not only legitimizes the fact of sufficient specification of the named concept, but also greatly reinforces this specification, causing its extensive relativization (by reinforcing the connotation relation with even "distant" concepts in the conceptual network). Thus, language is primarily an excellent tool for efficient use of the conceptual network. The crystal of concepts is more general than language in the denotational sense, which means that each name is matched by a concept, although there are some concepts (areas of the conceptual network) that are without names. It is also superior in the connotational sense, as language itself and the denotation relation between names and concepts are also a part of the conceptual network which is connotational by nature. In language, a definition is a certain substitute or a "model" of meaning through connotation. Here, the meaning of a given name is determined by the relation to other names. But, while a definition in language, however extended, consists of a limited number of words, a concept is basically "defined" by all other concepts, by the whole crystal of concepts. The very process of understanding a definition does not take place in the linguistic layer, but in the conceptual one. Contrary to appearances, definitions are rarely used to define completely new things which are not yet placed in the conceptual network. Usually, a definition aims to clarify a concept which is already well-positioned in the network. As no definition, even the most exact one, specifies precisely the defined object, it only indicates a conceptual area where this object is supposed to be. If in a given area there has already been a more or less crystallized concept, then, as if automatically, the definition begins to refer to it. However, if the definition finds a relative conceptual emptiness, then there are potentially a huge number of concepts which might correspond to the area delineated by the definition. Of course, this area can be narrowed down by specifying the definition, but still the number of potential "interpretations" is huge.

Let us take, for example, the following definition: "onelephant is a one-legged elephant". As this is a completely foreign formulation in our conceptual "tradition", we do not know what it is, without the proper context. So, we have no idea if we are talking about an elephant with three legs cut off, or an elephant with a genetic mutation for one-leggedness, or a fantasy creature, or something from the conventions of science fiction (the allegation that it is a formal definition and nothing can necessarily correspond to it is misplaced here, as in contrast to the names of language, there are no "empty" concepts; speaking elsewhere about such concepts, we mean either concepts that have been "dismantled" as a result of semantic analysis, or simply the names of language). Whereas, in the definition "Loxodonta africana is a species of elephant living in Africa", everything seems obvious, as we know that there are two species of elephants in the world, including one in Africa, and we now learn that this species is called Loxodonta africana. So, we see that the semantic autonomy of language is only apparent-originally the meanings appear in the conceptual network. Thus, the conclusion is: a linguistic definition, if it is to be sufficiently meaningful and unambiguous, must refer to a single concept suitably well-distinguished and specified within the conceptual network, located in the area delineated by the definition. There should be no other concept in this area.

The crystal of concepts is also more general than language in the sense of its primary nature. This is simply because language cannot do without its conceptual "lining", it does not mean anything by itself, it is an empty structure of names. And finally, the whole language, with its names and grammar rules, can be expressed by concepts, whereas the reverse relation does not hold: it is impossible to describe the conceptual network using language (of course, we mean a complete description, otherwise the present work would not make any sense). Language is a certain conceptual map within the crystal of concepts, a map which is relatively well-distinguished and relativized to other maps. It plays a very important practical function, coming down basically to the efficient use of the crystal of concepts as a whole (and, of course, to interpersonal communication). It does not possess, however, two fundamental features of the conceptual network: its all-embracingness and reflexivity (the fact that a "linguistic name" is also a linguistic name is not sufficient, as language is not semantically autonomous). It is simply impossible to go beyond concepts.

To put it even more simply, language is the part of the conceptual network which constitutes a rough reproduction (a map) of the whole network. Let us appeal again to the analogy with landscape, but making a reservation that this time the analogy is very general. Imagine that someone engraved a map of the landscape (the area 
considered) on a flat boulder. In this case, the map is both a part of the landscape (as the surface of the boulder), and a reproduction of the whole landscape (as a map). If that map contained the names of hills, it would greatly facilitate our orientation of the terrain, so that we could distinguish one hill from another and remember their identities. In the same way, language as part of the conceptual network, enables orientation and usage of the whole network. Of course, on the map, there would also be a point signifying the map itself and the boulder, on which it is located, similarly as in language there is the name "language".

It follows that the denotation function of concepts in relation to the names of language and to the "real facts" is secondary to the connotation function of the conceptual network and it is reducible to it. Thus, if for a moment we forget about the property of reflexivity and regard a concept as an "absolute" entity, we could say that ultimately only concepts "exist", without their designata, because the whole structure of the designation relation is also a part of the conceptual network. To avoid suspicion of solipsism, we must make it clear that we are not describing what the world is, but only what we can say about it, and thus we are taking in this respect a position similar to that of Wittgenstein. The names of concepts are secondary to the concepts themselves, because if we can imagine the autonomous existence of meanings (a semantic space), a system of signs devoid of meanings is nothing but a collection of properties allowing particular signs, determining their identification, to be distinguished. So we can go beyond the system of signs (in the direction of unnamed meanings) and demonstrate its derivative character in relation to the system of meanings. So, is it true that "everything is a concept"? No, because the concept is also a concept and it cannot validate itself. Thus, through the property of reflexivity, we close the circle for the third time and return to silence. For the third time we must realize that we incurred a debt from "the absolute truth", attributing to the concept a privileged place in relation to "everything else". We believe that it is better suited for this purpose than "matter", "spirit", or "monads", precisely because of its property of reflexivity.

Language is not autonomous because the reception of its statements does not depend on its inherent or absolute meanings, but depends on the predispositions and conceptual structure of the recipient. Any text, together with its underlying system of meanings, each time will be received in a slightly different semantic environment, by a slightly different crystal of concepts of a given recipient. Depending on the specific case, the text will be understood, not understood, or misunderstood (regarding the author's intentions), and all intermediary states are possible. We should also note that historical epochs, affecting all contemporary minds, change the perception of a given text (e.g., a literary one). In short, what meanings will be received from a text, depends on the cultural, historical, and psychological context of its recipient. The same applies to individual sentences of a language or even individual words. In fact, this applies to broadly defined language, i.e., including gestures, facial expressions, systems of symbols, and the rest of signals. Perfect communication, that is, the perfect agreement between sent and received meanings, is impossible, precisely because the transmission takes place in the linguistic layer, whereas the understanding of meanings goes on in the conceptual layer. And there are no two identical crystals of concepts. To put it another way, language serves to translate between two different crystals of concepts. As the correspondence between the conceptual network and language is far from clear-cut, the process of translation will never be perfect. Therefore, perfectly accurate communication between two entities is impossible on principle. Faithfulness and even the possibility of transmitting information between different crystals of concepts depends on their similarity, and that is why we believe that the understanding of this text is tantamount to the acceptance of its "truthfulness".

The problem emerges of what to do with other crystal of concepts (other consciousnesses). The absolutization of concepts and the conceptual network would inevitably lead to the conclusion that they too (like everything else) are part of our crystal of concepts, and that position is not far from solipsism. However, the main objective of this work is to demonstrate the lack of legitimacy of the absolutist approach. The idea of the conceptual network was adopted as a temporarily useful model, which will be renounced immediately after we reach conclusions that are interesting to us. Regarding the question of our inability to prove-within our mind-the external world and other consciousnesses, despite their objective existence, it seems that the idea of the evolutionary trap of consciousness, discussed in the section on the internal philosophy, might help to answer it.

Let us return for a moment to the denotation relation "concept—an element of language". In this view, it is not a typical denotation, as the concept being denoted (we assume a symmetry of the denotation relation) is unquantized, continuous, and not exactly defined. This property translates into the relation itself. It is also not entirely clear and defined. So, we could reserve the name "denotation" for situations when the denoted element, the denoting element, and the relation itself are strictly defined, and we could assign a completely new name to 
the described relation. We could also suitably extend the range of the name "denotation" to make it cover both types of relations. However, an intermediary solution seems to be the best: we could add the adjective "apparent" to the noun "denotation". "Apparent denotation" is a good term also because, as we emphasized, it is secondary to the connotation relation of concepts, exactly as the denoting element, i.e., the language, is. Thus, in this work, when we speak about the denotation of concepts by elements of language (or the other way round), we mean the apparent denotation.

The concepts have meanings through connotation, which is why we can tolerably efficiently use them (and the corresponding structures of language) only in the centre of the conceptual network, where there is a considerable intensity of the semantic field. When the semantic location of a concept, determined by its connotations, becomes insufficient, the meaning of the concept dissipates in our consciousness, and escapes the sphere of language. This can occur when we are close to the periphery of the conceptual network, where poorly marked concepts border with the semantic void, and when during semantic analysis of the conceptual network we deprive a given concept of some of its previous connotational references. In the latter case, although it seems to be a disambiguation of a concept through precise delineation (=limitation) of its relations, we end up with a concept different from the original, if not with an empty name.

The concept of justice (in the sense of administration of justice) can be analytically reduced to the concepts of prevention and retribution. The point is that we cannot give any specific reason for the administration of justice, which would not fall into one of these two categories. We mete out justice either to discourage breaking the law by a particular offender and other members of society, or we want to take revenge in the sense of "an eye for an eye”. But justice so understood has little in common with our sense or ideal of justice. After all, each of us intuitively feels what justice is, and this feeling is not in any way invalidated by the conceptual analysis of justice. It is simply that this analysis, by attempting to disambiguate the concept, breaks its connotational ties with our feelings, emotional assessment, moral evaluation, etc. The end product of this analysis is simply another concept under the same name. We will call this process analytical desemantization. Its essence is somewhat similar to Heisenberg's uncertainty principle: the more analytical power we put into analysis of a given concept, the harder it will be for us to locate it semantically, as we will break more of its semantic ties with other concepts.

Wittgenstein's conception (we still refer to his Tractatus Logico-Philosophicus) is in fact a conception of absolute meaning of language. The names of language which have designata in the world are meaningful by themselves, whereas other names are empty. Nonempty names denote objects in the world, but this does not explain where the understanding of their meaning comes from. The connotation relation is impossible because of the quantized nature of language. As quantized meanings allow for a relatively very small number of possible combinations, it is unfeasible to create such rich range of meanings with them, as is characteristic of the human mind. And, more importantly, the lack of continuity between meanings assertorically excludes any contact between them. In this view, names of language have meaning in a primary, a priori, non-confrontable way. This is a picture of language as a rigid, inflexible structure, which does not undergo any changes or fluctuations. And this obviously contradicts our experience. Leaving aside the question of unnamed meanings, we everywhere encounter instability and a kind of indeterminacy of meanings of the names of language. In addition, the absolutism of meanings stands in contradiction to the ongoing emergence of new concepts from existing ones. Finally, the idea of meanings which are meaningful by themselves is "inelegant", and such meanings resemble completely isolated monads. A "mechanism" of any relations between these meanings remains a mystery. This view is, apart from fundamental objections, intellectually barren, as we still do not know anything about the nature of meanings (certainly, it is not a denotation of the facts of the world, as it does not say anything about understanding of meanings), which as a result of their absolutism are not amenable to analysis. The theory of relativity of meanings seems to better describe the set of phenomena accessible to us, than Wittgenstein's philosophy of language which leads to semantic absolutism. One should emphasize that in Philosophical Investigations (Wittgenstein, 1999), Wittgenstein significantly changed his views. He moved on to positions closer to those presented in this work, but his system lost all its momentum, consistency, and clarity.

To sum up, language is a secondary entity in relation to the conceptual network and that is why we may regard it as a relatively well-defined part of this network. Language greatly facilitates the use of concepts and therefore adds to the dynamics and development of the whole network. This "function" evokes a subjective impression of designation relation between concepts and the names of language (as well as "material facts"), although this relation is secondary to the connotation relation between concepts. 


\section{The Absolute as a Delusive Reference}

One of the main messages of this work, which has been already repeatedly formulated explicitly, is the statement that it is impossible to say anything about the world in an absolute way. The absolute truth would be something with an autonomous meaning, understandable by itself, something independent of any contexts and references. The above definition is an empty name, to which nothing "real" or sensible corresponds. From the stated above, it follows that something like autonomy of meanings is not possible (assertorically). We return to this point constantly, as the entire history of philosophy (in particular ontology) followed a familiar pattern: you assign an absolute meaning to a concept and this way create the axiological foundation for a description of the world. Such presuppositions were necessary, if we wanted to express in an absolute way universal truths about all the phenomena accessible to us. And yet, despite attempts lasting thousands of years, philosophy has not been “completed”. A description of any, even the most current philosophical system, ends in any careful study with a list of reasons why the system is in fact unacceptable. There are usually two kinds of allegations.

First, there were always some phenomena which could not be subsumed under this absolutized concept (e.g., the concept of matter, spirit, monads, etc.), and thus lacked the property of universality. It can be argued that the whole sphere of consciousness is a derivative from physiological brain functions, and therefore has a material basis. However, it is impossible to maintain that the entire human mind "is" matter. On the other hand, a consistent solipsist would have to agree that his own ego is also an illusion, like the rest of his impressions. Thus, consistent idealism is also not sustainable. As for the monads, they lose general and abstract concepts.

Second, when you examine a philosophical system based on the preliminary absolutization of a concept, you always come in your analysis to that concept in the end, and then not only the attributes of its absolutism, but also the concept itself dissipates into the semantic void, leaving only an empty name. Earlier we gave an example of this process regarding the concept of matter. Generally speaking, the act of absolutization of some part of the conceptual network and a reflexive reference of the rest of the network to this part, eventually leads to a contradiction. This contradiction stems from the fact that in order to talk about something as a whole, you should be outside of it, at some higher level. However, if we talk about everything (as ontology does), we obviously have to do it from the inside. If there were some external point of reference, this "everything” would not be a true "everything", as there would be something outside it. This external reference point, impossible by its very nature, is the only one from which the absolute truth could be proclaimed. Therefore, the absolute truth is a self-contradictory concept, and this self-contradiction can be found in every philosophical system. It is the same with the system presented in this work. We took a concept as our starting point, and despite its many positive philosophical attributes and despite constant appealing to the property of reflexivity, we have come to some kind of its absolutization. Some kind of meta-level emerged which is external to "everything"; it is an extension of the axes of meanings beyond the crystal of concepts into a "delusive" semantic space. However, this procedure differs from those mentioned above, as the said contradiction has been incorporated as the first axiom of the system, which has been an inevitable consequence of going beyond silence.

This operation frees us from the fear that we will find this contradiction at the periphery of our system, destroying, to our dismay, its seemingly monolithic edifice. Of course, this way we delegitimize our system explicitly in the absolutist reference (but it is better than having "hidden" contradictions). Importantly, this delegitimization applies to the absolutist reference in general, and this is the primary objective of this work. In this reference only silence remains, and this is another closing of the circle, another return to the statement expressed in Section 1.

So if we want to speak "the truth and nothing but the truth" consistently, we should remain silent. This is the first level of cognition. However, this is obviously not satisfying for us. We want to be able to say something about the world, even if it will have to be, to some degree, non-legitimate and self-contradictory. So did philosophy, and thus we proceed in this work, for there is no other option. However, the conception of the conceptual network has some merits in comparison with other systems. First, it automatically leads to the awareness of the state of affairs outlined above. Like early Wittgenstein's conception, it points to its own lack of legitimacy, although from a slightly different position. Second, it has the properties of universality and reflexivity. The former's value for a philosophical system is obvious. The latter allows us to reduce contradictions in the pronounced (almost absolute) truths to the role of an axiom of the system. This way, we reach the second level of cognition - the conceptual network. Of course, this is not the only possible second level; potentially there are probably an infinite number of them. It is constituted by every philosophical (ontological) system. Nevertheless, 
a "good" system on the second level should reveal its own non-legitimacy and point to the first level as the "end of cognition". The third level is constituted by the "everyday world", by all the phenomena given to us in their immanence, in everyday life, in its obviousness. Between the second and third level, there are: natural science, humanities, mathematics, logic, philosophy, and religion. The division into levels, as any division of this kind, is completely arbitrary; the transitions between them are continuous. This division does not reflect any invariants or absolute categories; it is a practical operation which aims to facilitate "moving around" in the discussed area. In particular, the truth (and reasonableness) of claims depends largely on which level they are made. There may not be any real contradictions, if we pronounce seemingly contradictory, or even opposite statements on different levels. Therefore, for each claim we need to say what level it is made at, and when we see a contradiction in some reasoning, we should consider whether it is not only apparent, as it has occurred between different levels. A simple example is the fact that we are writing these words, although we consider silence to be the "absolute" end of cognition. Many more of this kind of misunderstandings may occur between the second and third level. In fact, the situation is even more complicated, as we can distinguish an infinite number of sub-levels which seamlessly transform into another, and often the meaning of claims is also relativized to them. This problem results from the connotationality of the conceptual network and can only be partially overcome.

As we have already mentioned, all ontological systems have relied on distinguishing some fragment (a concept) of the conceptual network, its absolutization, and making it primary in relation to all the rest of phenomena. This meant that "really", "in a deeper layer" all these phenomena were designata of that concept. The whole variety of accessible phenomena would then be some illusion in the sense that it would be a secondary quality in relation to the unity represented by that distinguished concept. Let us call that concept $\mathrm{X}$-such that the scheme of almost any ontology could be written in a simplified formula "everything is X"-Alpha. Thus, Alpha is an absolute entity, indefinable (implicitly, as not all creators of philosophy have been aware of it). That is, at least in principle, the idea - an intuitive impression of Alpha's "truthfulness" is supposed to make any other validations unnecessary, even unacceptable. We are to take the legitimacy of Alpha at face value, and any kind of its verification is an absolute taboo. It was the same with all kinds of religion, which is why religion resembles ontology so much.

Of course, in the history of philosophy Alphas differed among themselves in their level of abstraction. From the simple ones, such as the four elements, matter or spirit, they evolved over time to more sublime ones, such as the will, monads or "neutral unity" in Russell's neutral monism (Mach, 1976; Russell, 1975). The claim that "everything is Alpha" was not always so categorically formulated, for example, in early Wittgenstein's philosophy (Wittgenstein, 1961). Besides, the above analysis applies only to the "positive" ontologies, i.e., those telling us what the world is. Early Wittgenstein's philosophy falls into this category only partially, although literally it distances itself from any kinds of "positivity". Imperfection of Alphas taken from everyday language manifests itself still in another way. Making an Alpha out of a concept taken from everyday language is based on transferring this concept, together with its name, to a philosophical system, because of this system's "conceptual situation", which is close to the original "semantic context". However, part of the semantic environment of the transferred concepts is (necessarily) inadequate to the new conceptual situation. And yet, if this transfer is to be fruitful, that is, if it is to bring something new to the system, we have to use other semantic relations of the transferred concept than those that were the reason that it has been transferred and made an Alpha. Unfortunately, with the change of the semantic context, the identity of the concept itself also changes. It is no longer the old concept from everyday language, and it has been also poorly "grounded" in its new environment. Therefore, it is much more susceptible to analytical dismantling and subsequent desemantization than the original concept. Erroneous equation of both concepts on the basis of their shared name often leads to the use of too far-reaching analogies, as in the discussed example below of discovering new lands and discovering mathematics (or inventing machines and inventing mathematics). Unfortunately, most of this type of non-legitimate analogies is much less obvious.

The need for absolute knowledge is, of course, most understandable for psychological reasons. Searching for a firm foundation of universal truths has always accompanied the human cognition. Unfortunately, this cognition has gradually deprived humanity of the Absolutes. The history of exact and natural sciences, and the philosophy related to them, is a series of gradual deabsolutization of everything. From its very beginning, modern science has forced every intellectual to choose between cognitive honesty and a psychologically comforting worldview. The Middle Ages were in many respects a happy time for intellectuals. Later, the two mentioned options (i.e., cognitive honesty and psychological comfort) could never be reconciled. At that time, the image of 
the Universe was very favourable for humans. There was the centrally located Earth, the Sun which revolved around it, providing light and warmth, the Moon which illuminated the darkness of night, and the sphere of fixed stars, showing the way to wanderers. Beyond that sphere was Heaven, the seat of the Supreme Being, whose close attention was focused on the centrally located Earth and its inhabitants. It was a small and cosy Cosmos, created for human beings and favourable to them in every respect. The central location of our planet was equated with the central importance of Humans in the Universe. The Copernican revolution shattered this crystal image. The vision of the world, regarded in the Middle Ages as absolute and certain, was demolished. For the first time, human beings were forced to relocate the importance beyond themselves, to abandon some Absolute. After Copernicus, there was Kepler, who, on the basis of observational data, decided to replace the circular planetary orbits with elliptical ones, although a circle had been considered a perfect figure since ancient times. Subsequent discoveries radically changed the medieval view of the world. Let us look at today's image of our Universe. Billions of light years of vast terrifying void filled with relatively rare stars and planets, signifying nothing, and serving nobody. And human beings, created by blind chance in the lottery of evolution, inhabiting a small planet which orbits one of the billions of stars. Even the picturesque Biblical finis mundi has been replaced with gravitational collapse, the thermal death of the Universe, or the Sun turning into a red giant and completely burning up the Earth. Thus, science has replaced the only possible, perfect Universe being at the service of Humanity, with a random Universe, filled with billions of light years of senseless emptiness.

It is not only the Cosmos as a whole that has been deprived of the mark of the Absolute by science. Physics questioned the semantic autonomy of many concepts taken from everyday language that are seemingly so obvious and "tangible". Newton challenged the absoluteness of the distinguished ("stationary") reference frame in space; Einstein questioned the very space and time. Thus, even such fundamental elements of our world are no longer a solid basis for our intellect; even they are no longer undeniable and indisputable. Quantum mechanics negated our conventional notion of causality. In addition, matter has suffered desemantization by physics. At the moment, it is impossible to establish a boundary between a "real" element of the world, and that which is only a mathematical model, a creation of our mind. Thus, even the absolute boundary between a concept and its designatum has been negated. It is difficult to feel intellectually "safe" in a world deprived of any absolute basis, where nothing is certain, and all is relative.

The advancing process of deabsolutization has also affected humans. Instead of the creatures who were made in the image and likeness of God, we now are a biological species with apes as the closest living relatives within the tree of evolution; and the immortal soul (what is left of a soul, when we "amputate" its consciousness?) has not survived the destructive (for the Absolute) force of science either. Radical changes found in the mind after brain damage, great progress in the study of neurophysiological mechanisms of perception as well as of the molecular basis of memory, have left little space for spirit in its immanence, or for autonomous and undivided consciousness. We can give some more examples, such as the deabsolutization of logic (set antinomy) (Russell, 1975), the deabsolutization of formal mathematics (Gödel's proof) (Barrow, 1992), the denial of a strict determinism (which was the Absolute of the pre-20th-century physics), as well as the denial of the "absolute" phenomenon of life (e.g., a "life force") (the transition from holism to reductionism).

The logical consequence of the shown route appears to be a demonstration of non-legitimacy of any meanings in the absolute sense, the denial of existence of any truths which are supposed to be valid regardless of reference. In this view, even Pascal's thinking reed turns out to be an illusion-no absolute knowledge can be given to us. Here we find also a complement for the Occam's razor- there is no need to assume that anything exists (in the absolute way).

Of course, the claim that the absolute truth does not exist cannot be the absolute truth either. You cannot make reasonable claims about the existence of designata of semantically empty names, and even more-to make claims using these names. Only the system of relational and relativistic truths is accessible to us. We build the system here on the basis of the concept of a conceptual network, but certainly other systems are also possible. The important thing is to recognize the relativity of truths at the beginning of each system.

The cognitive chain of philosophy can be presented as a cone. Its top would signify an infinitely clarified internal truth, which philosophical systems strive for (asymptotically). The further away from the apex to the circular base of the cone, the less the systems are clarified, and the more loosely the network of concepts covers the world of phenomena accessible to us. Let us note: there are no philosophical systems which are completely "true" or "false", there are only systems which are more or less specified, adjusted to the spectrum of phenomena accessible to us. A philosophical system claiming that everything is a redcurrant jelly is not completely and abso- 
lutely false. It is simply that in our conceptual network this claim is so poorly justified by the connotation relations of the concept of "fruit jelly", and in particular, "redcurrant jelly", that we reject it, without hesitation, as false intuitively. However, between the said system and, for example, materialism there is only a quantitative difference in the "degree of truthfulness". The concept of "matter" is a lot more associated with something that is everything, although its legitimacy in this role (as in the case of any other concept) will never be absolute.

As the crystal of concepts developed and the meanings "got denser" on its edges, in the history of philosophy, it was possible to make a better description of "everything", although it is still far from the apex of the cone. In this analogy, different sides of this cone correspond to different aspects of reality (or different fragments of the conceptual network). Philosophical systems are circles, or rather ellipses inscribed in this cone, and the planes on which these ellipses lie, are not parallel to the base of the cone. This means that a given system is better defined in a certain aspect of reality than in others. In particular, in this view, there is no point in saying which is truer: materialism or idealism. We argue that they are placed at a comparable distance from the apex, but their ellipses are steeply inclined in opposite directions, which gives the impression of the complete opposition of these systems. Of course, both materialism and idealism are not homogeneous. These systems have evolved over time, gaining new views and clarifications, and gradually they moved closer to the apex of the cone. This is also true for other ontological systems. All philosophy can be described as a tangle of ellipses inscribed in the cone, tilted in different directions at various angles, at different distances from its base. If we assume that this base corresponds to the everyday, unreflective view of the world, then, above it, there are all kinds of animistic concepts, the idea of the four elements, and other ideas, taken directly from the conceptual map of everyday life. In our view, the ellipses of Russell's neutral monism and Wittgenstein's philosophy of language (Wittgenstein, 1961) are closest to the top of the cone. We present the current system because we think that it goes a step further, that it is an ellipse (or perhaps a circle) that cuts the cone relatively closer to its apex. This alone shows that one can go further. The only limitation is the crystal of concepts (or its equivalent in the future philosophy) that we have at the moment. As it develops, under the particular influence of science, we will be able to specify more and more perfectly the description of the "internal" world of phenomena accessible to us. Finally, to avoid any misunderstanding, we have to make it clear that obviously the analogy with the cone is a simplification, which helps to illustrate a certain idea. Too far-reaching implications of this analogy may be non-legitimate. In particular, the two-dimensional space of ellipses can only very imprecisely be compared with the semantic space of philosophical systems having a large, but unspecified number of dimensions.

Philosophy has not developed any objective criteria of evaluation or selection which would regulate the "promotion" of various concepts to the position of Alpha. The only determinant of the value of ontologies (their subjective truth) is the intuitively felt truth of a given Alpha and its "grounding" in the conceptual network. So, why do we want to add a concept to so many existing Alphas? First, speaking half-jokingly, because of the subjective sense of truth of this approach. There are, however, more important reasons, which we have already mentioned. The concept has two properties which are not possessed by other Alphas. The first is versatility. The concept includes all the phenomena accessible to us on all levels of hierarchy. Matter has nothing to do with the vast realm of spiritual life, dreams, joy, suffering, intuition, hunches, or nightmares. Spirit does not explain why the world is pacing steadily along the route measured by natural sciences; it does not explain the geniuses' surprise at the fact that the Universe can be described with relatively simple mathematics. Furthermore, if the material world is an illusion, then we must also regard consciousness as an illusion (if we consider both just a bunch of sensations). Besides, in the old dichotomy of spirit and matter, it is an aesthetic flaw to regard any of them as more primary. Lastly, when we think of spirit, matter, will, monads, and elements, we are struck by the arbitrariness of promoting a given concept to the position of Alpha. We could conceive of other concepts, e.g., the fluctuation of nothingness, which are equally suitable for this function. It seems that the main criterion was the need for a simple and understandable world. In this view, we equate extreme simplicity (we cannot go beyond the network of concepts which are categorially identical) with infinite diversity (multiplicity of phenomena designated by concepts). And finally, the development of the conceptual network of humanity has made the above-mentioned Alphas seem somewhat outdated.

The second, earlier discussed property of the concept is its reflexivity. Such an element as matter or spirit, after promotion to the position of Alpha, becomes the Absolute, a primary concept, a perfect entity, of which any analysis is prohibited by a powerful taboo. Subscribing to materialism, idealism, or any other ontology requires us to accept our Alpha on faith, at face value, and to place it in some corner of our mind and never come to it again. And again, apart from an intuitive and aesthetic objection to such modus operandi (philosophy is not a re- 
ligion, or at least it should not be a religion), even logical constructions that contain such primary concepts are failing. Physics made the concept of matter melt in equations, field intensities, wave functions, and probability distributions; matter is slipping through our fingers. There are similar problems with the spirit, for what about other consciousnesses? What about the spirit's dependence on matter (e.g., changes in the psyche after brain damage)? The concept of the concept is different. The concept (the crystal of concepts) contains the whole Universe of phenomena, and it also contains itself, and by this, it indicates the non-legitimacy of such a system. Thus, the concept is Alpha but only to show that it cannot be Alpha.

\section{The Conceptual Network and Logic}

In Section 3, we outlined the relationship between the conceptual network and language. Now, we will deal with the mutual relations between the conceptual network and logic. We have already said that we are not going to argue by means of logical analysis, but will instead try to create a "conceptual vision" that fits into the conceptual network of the reader. This means a denial of logic as a universal research tool in philosophy. Below, we will try to give reasons for this position.

What is logic? Logic provides rules to predicate truth or falsehood of certain sentences (or, to follow early Wittgenstein, it simply consists of these rules). These sentences contain a relation (or relations) between some elements, or they simply define certain properties of these elements. The elements can be composed of individual entities or their sets (categories). Logic seems to be an absolute and autonomous entity, like mathematics. Its principles are so intuitively clear, obvious, and so unsusceptible to any doubts regarding their legitimacy, that we tend to believe that we could deny anything, but not logic. Wittgenstein in his Tractatus Logico-Philosophicus absolutized logic to the extent that he treated it not as a subject of cognition, but as the structure of cognition, or even more- the structure of the world. In his early system, logic was an inherent property of language that set the limits of our world. Even more than inherent-it was the only possible, reasonable, and necessary property.

Nevertheless, we will try to demonstrate that the absolute legitimacy of logic is an illusion. Let us take a most simple statement of the type "A is B", for example: "a rose is red". This sentence is intuitively obvious, perfectly clear, and understandable for everyone. But is it really absolutely unambiguous? Let us analyse its elements. Let us start with the word "rose". We could apply the bald man paradox to its referent, that is, to the real plant. If you take one atom out of a rose, it still remains a rose. The same is true, if we take two, three or four atoms out. But the process of consistent subtraction of atoms eventually leads to an entity which is no longer a rose, and we cannot say when exactly this happens, we can determine it only arbitrarily. Of course, "a rose" is not the name of a single object, but of a certain category. The above analysis serves to show that we cannot determine at which point the rose being stripped of subsequent atoms leaves this category. Thus, the concept of rose is underspecified spatially (structurally). In order to specify it better, we would have to-in our definition of the rose-specify the position of each atom (although because of quantum mechanics, that would probably not be enough). This, however, would give us a definition of a single material object, and not a category of objects which are called roses. This proves that the category of roses does not exist ideally (absolutely), in every sense of this word. In this situation, either we base logic on single individuals, abandoning the category (and this, at a lower level, also leads to deabsolutization, as we will show in a moment), or we accept the fact that logic operates on elements which are not perfectly defined.

In fact, the example given above is only a single aspect of a more general phenomenon. Let us move on to other aspects. A rose as a mature plant grows from a seed (through the seedling stage). It is impossible to determine the exact moment of transition between these stages, that is, at which point we stop calling it "a seed", and start calling it "a rose". Thus, a rose is underspecified in time (ontogenetically), which is in fact related to its structural underspecification, but of a different kind than in the previous example. A rose as a plant emerged in the course of evolution through a whole series of transitional forms, from plants that were not roses yet. This is a phylogenetic underspecification of the concept of rose. And finally, we should mention the most important, categorical underspecification, which in fact contains all of the previous ones. Now, we can imagine a continuous series of objects (such that two adjacent objects are almost identical, but differing only very slightly), which are more and more different from our standard rose. In this series, there is somewhere a point where we most certainly cannot call the object a rose. Of course, this "series" is not one-dimensional, but potentially has an infinite number of dimensions (that is, the features in which the adjacent objects may differ). In such a series, we 
can only arbitrarily determine how far the category of a "rose" reaches. Here, it turns out that such a seemingly clear concept as the concept of a "rose" is not defined absolutely, but just intuitively. The impression of clarity and unambiguity of this concept comes from the fact that usually, in the set of all phenomena accessible to us, there is a clear divide between roses and other objects (a gap in the continuous series of variation), which makes it easy for us to distinguish the class of roses as a separate category. It is an empirical property, pragmatically useful in this case, but not able to prove the absolute specification of the category. In many cases, however, for example in philosophy, there are not even such pragmatic "relatively good" specifications of concepts.

It is even simpler to deabsolutize the exactness of the name "red". In a continuous spectrum of colours ranging from orange to violet, we can only arbitrarily indicate the red colour. Moreover, research has shown that the range of the names of colours is an interindividual variable (i.e., different people draw slightly different boundaries on the continuous spectrum of colours, e.g., between red and orange) and, to a greater degree, an interlingual (i.e., different ethnic languages differ in this respect) and intercultural variable. Besides, even if somebody can easily identify certain points on a red-violet spectrum as definitely red, or definitely violet, they are simply not able to categorically determine other points as belonging to one or the other category. We lack names for the continuous - and therefore infinite-spectrum of phenomena. Thus, logic using names is able to describe the world only in an approximate way. In addition, the meaning of the verb "is", which is well-defined in some semantic areas, blurs on their peripheries. It is possible to "be" an object, category, concept, thought, idea, feeling, thing, fantasy, but it is also possible to "be" in the sense of "exist". In each case, it is a different kind of being (with different attributes), and in fact each of them should have a separate name. After all, a concrete tree "exists" in a different way than a moral principle or category does. But this still is not a solution, as the boundaries between the said types of "being" are not absolute. There is a seamless transition from one type into another. For example, theoretical physics often struggles to determine whether a given concept refers to a "material object" or a "mathematical creation", and it turns out that in many cases it is impossible to make this distinction. For example, there is the problem of the "real" existence of atomic orbitals, described by wave function. The fact that it is impossible to separate exactly different modes of existence (for example, we cannot draw a sharp line between real persons and those encountered in dreams, until we are sure that we are not dreaming or hallucinating at that moment, and this is not entirely possible) leads to the conclusion that, in principle, they are vested with one kind of existence, that in this respect all the phenomena accessible to us (objects, ideas, categories) have "equal rights". In our view, this corresponds to a flattening of the hierarchy of concepts in the conceptual network to categorial unity.

The examples given so far have dealt with categories and general entities. But the conclusions also apply to individual entities. Let us take the sentence: "John is in this room." If we know John and we are in the same room, then we can easily state whether this sentence is true or false. However, if we deal with John as we did with the rose, some problems arise. Let us cut his hair and fingernails. John is still in the room. Let us amputate his hands and feet. So horribly disfigured, John is still John. However, if we managed to keep John's isolated brain alive, calling it John could raise some doubts. But let us assume that driven by humanitarian instinct we call it John. We could then carry out corpus callosotomy on this brain (cutting its corpus callosum). As a result we get two, largely isolated consciousnesses. Is it still John, or are there two Johns now? Of course, we can further disassemble the brain, until we get something that most certainly is not John. Thus, we see that by mechanical dismantling of John, we can also break him down semantically. And what if John's identity relies on his consciousness? By all means! It is a known fact that severe damage to the brain can turn a saint into a murderer, and a genius into an idiot. What happens, however, if we do not injure John's brain radically all at once, but we cut out a single neuron at a time? When will John end and someone else emerge? Thus, we see that we are forced—having first apologized to the so brutally treated John—-to recognize that logic is not legitimate in relation to individual entities either.

Going further, the meaning of any sentence (also a sentence constructed according to the rules of logic) depends on its context. Of course, the semantic context can be, like any concept, much better defined. But a sentence built within a language will never gain an absolute univocality, i.e., it will never be possible to attribute to it a strictly specified area of the conceptual network (which is not univocal either). Logic retains more or less its validity (i.e., the elements and relations described by it are satisfactorily specified) only in certain regions of the crystal of concepts. These are the fragments of the conceptual network where the meaning of concepts is sufficiently well established through relation with other concepts, where the conceptual network is dense, and the intensity of the "semantic field" is large. Philosophy does not belong to these regions. Therefore, logic is helpless 
in dealing with philosophy. If syllogisms are to be applied to names which are little more "meaningful" than empty names, if sentences of logic are to use concepts bound together by very poorly specified relations, then their content turns out to be gibberish. Therefore, any consistent logical analysis leads to complete desemantization of any philosophy, and that is why the early Wittgenstein and the logical positivists claimed that statements of philosophy are meaningless, although they mistakenly contrasted the sentences of philosophy with the sentences of science-from the absolutist point of view all of them are meaningless, whereas the sentences of science are undoubtedly better defined, which is a measure of their reasonableness in the presented view.

There is, however, no sharp boundary: science transforms smoothly into philosophy. Just as there is neither absolute truth nor falsehoods, there are no sentences which are completely meaningful or totally meaningless. We can construct relatively more meaningful sentences within conceptual maps with a high intensity of the semantic field, containing well-defined concepts. In addition, the opposition of truth and falsehood turns out to be illusory. Speaking figuratively, we could say that while classical logic (implicitly absolutist) attributes to any meaningful sentence the value of 1 (true) or 0 (false), conceptual relativism leads to a situation where any possible claim can take any of the intermediary values, but never 0 or 1 . There is only a relative measure of truth, i.e., one can say that something is closer to truth than something else, which in turn will be determined by the degree of coherence of a given statement with the conceptual situation in which it was made. And, most importantly, this degree of truth is nothing absolute, but entirely depends on the above-mentioned conceptual context. Therefore, this is a totally unacceptable situation for classical logic. In opposition to Wittgenstein, we claim that logic is not entirely opposed to all empirical claims, that it is not only a manifestation of their structure; on the contrary, we believe that its rules belong to exactly the same category as the sentences describing the world of phenomena.

It is possible to reconcile somehow with the fact that no sentence is absolutely true. But is it not an exaggeration to say that there are no absolutely false claims? Let us consider again the sentence: "John is in the room." We enter the room and we cannot see John. Have we not made an absolute falsification of that sentence? No, because it all depends on the context. First, John may be in the said room in his thoughts. Second, if we are believers in "panjohnism", we find that John is basically everywhere, and therefore also in the room, although we cannot see him. Third, let us suppose that a piece of John's epidermis, just a few cells, is in the room. Now, let us apply the bald man paradox à rebours. Let us add one atom to this little piece of skin. This is not John yet. However, if we are sufficiently consistent in adding these atoms, then soon we will have John's leg in the room, and eventually - the whole of John. Thus, there was the beginning of John in the room. Fourth, if on average John spends 40 per cent of the time during a day in the room, we could say that John is in the room with the probability of 40 per cent. And fifth, we can hallucinate that John is not in the room, although in reality he is there. The probability (degree of truth) of conclusions cannot be greater than the probability (justification) of premises. Therefore, if we are never 100 per cent convinced about realism, we cannot with all certainty make any claims on the basis of our senses. We believe that further examples are not needed.

In a similar way, we can undertake to justify so ridiculous a claim as "everything is redcurrant jelly". First, some part of the world (admittedly rather small) undoubtedly is redcurrant jelly. The whole world is continuous which perfectly corresponds with the continuity (or runniness) of jelly. The jelly is dead, like the Universe, but as a substance containing starch (an organic compound, after all), it carries within itself some germs of life. Solid substances are very dehydrated jelly, and liquids are jelly with the proportions of ingredients shifted against starch. The differences between various solids and liquids are only illusions of our senses, whereas fire is the redness of the redcurrant released from the jelly. Therefore, everything is redcurrant jelly, but our senses are too imperfect to see it. Of course, we do not identify ourselves with this silliness, but are not the arguments of many philosophies close to it? Thus, we have to admit that there are no sentences which are so ridiculous as to be absolutely false. We could only intuitively determine the degree of their probability, and we could do it only in a specific semantic context (a sect of redcurrant jelly followers would probably regard the above sentence as very probable).

Generalizing further, we can say the following: the world eludes logic, as logic is a form of language. By carrying out operations on names, logic cannot properly describe the crystal of concepts because it is limited by the imperfection of mutual correspondence between the crystal of concepts and language, as was discussed in Section 3. Nevertheless, there are some regions where concepts exhibit a high degree of specification (although not absolute, of course) and they are well-distinguished "building blocks" of language, which can be used by logical syntax to build statements or syllogisms. In such areas, logic is quasi-legitimate. These include most of the ex- 
act/natural sciences and "everyday sphere”. The sentence "a rose is red" is in colloquial speech quite reasonable, that is, one can well predicate its truth or falsity. The meaning of words is defined well enough to intuitively perceive their sense as seemingly absolute and autonomous. However, regarding philosophy, this quasi-legitimate logic loses its validity almost entirely.

Contradictions at the level of logic differ from those at the level of the conceptual network. The latter arise from imperfect agreements between various areas of the conceptual network, which we discussed in Section 2. Therefore, they are not "sharp" or quantized like contradictions within the language of logic, but grow gradually with the increase of "distance" between the confronted areas or maps in the conceptual network, from which we take the concepts we want to refer-to each other. A straight-line tangent to a circle is a good analogy. Contradictions within language emerge when inaccuracies of agreement in the conceptual network are large enough to be registered by discrete names of language. Then, "quantity turns into quality". However, the contradictions often do not appear clearly at the level of language due to incomplete unambiguity of correspondence between concepts and names of language. Without this ambiguity, language would not work, as it would constantly fall into contradictions. Besides, we would need too many names of language to describe concepts differing from one another only by fine nuances. Finally, some ambiguity in the designation relation is unavoidable due to continuity of the conceptual network and quantized nature of language. Thus, this is another reason why contradictions within logic must exist. We can say even more: without these contradictions, language, and therefore logic, would not work at all. An analysis of any conceptual system, and in particular a philosophical system, reveals many contradictions. It is no different with this system. That is why we need to emphasize once more that the presented view is not supposed to appeal to the reader by its logic, but by its "conceptual vision" which is to evoke in his or her mind an impression of truth (or at least intellectual satisfaction). If someone, after thinking about this work, does not agree with its ideas, this means that they have not understood them, that is, he or she is a person mentally different from the author. Because, in the discussed context, understanding of the presented vision is equivalent to its acceptance. In the absence of this acceptance, putting forward any arguments for or against it is pointless-we can only recognize a qualitative difference in the structure of our crystal of concepts.

In logic, there is a well-known distinction between sharp and vague concepts. However, according to the presented view, perfectly sharp concepts do not exist. In logic (and mathematics), such concepts as a functor, relation, integer, even parity, tautology, denotation, and negation are only approximately sharp (despite the subjective impression of their absolute clarity and univocality). Let us take the concept of the even parity of a number. Is it also a fuzzy concept, like those of the rose and John? Yes, although in a much lower degree, not registered by our mind. An even number is an integer divisible by 2 . The concept of number 2 was formed in our minds by the abstraction of a certain common property of different configurations of stimuli which appear to us as, for example, two stones, two fish, two apples, etc. However, if we subtract subsequent atoms out of one these two apples, then we get a continuous transition between the two apples, and the amount of apples is slightly less than two. If we have two stains: one red, and the other almost-red (only infinitely a little different from red), then how many red stains do we have: one, two, or one and a fraction? Moreover, the concept of even parity is defined by other concepts (e.g., divisibility), which also are not absolutely sharp. Thus, the sharp meaning of the concept of number (or even parity) is only an introspective impression of our mind. It is a similar situation with the tautology mentioned earlier in this work: "Everything is Everything" (in the sense: the totality of phenomena is the totality of phenomena, and not in the sense: each phenomenon is any other phenomenon; the latter is obviously not a tautology) also cannot aspire to perfect sharpness. In philosophy, even truisms do not have absolutely sharp meanings. As for the latter, the concepts used there are very far from sharpness. A philosopher demanding from philosophy the exactness of formal logic is like a person looking for his or her lost property under a street lamp: they use tools they would like to use, regardless of whether these tools can be meaningfully applied to philosophy.

Finally, let us consider our conception's relation to three logical paradoxes, namely the liar paradox, Russell's antinomy of classes and Gödel's proof. It is best to present the first in the form: is a man who says: "I'm lying" telling the truth or is he lying? It is easy to see that both answers lead to contradictions. The antinomy of classes is expressed in the question of whether the class of all classes not being their own element is or is not their element. Since in this case both alternatives also lead to a contradiction, Russell, saving the unity of logic, introduced the theory of logical types (Russell, 1908). In all probability, nobody was fully satisfied with this theory, as it bypasses the problem, instead of solving it. Gödel's proof shows that in mathematics (and specifically—in a certain type of formal logical systems), there are statements which can neither be proved nor disproved, as such 
formal systems incorporating all rules of mathematical proofs, applied to themselves, lead to a contradiction. As a matter of fact, Gödel constructed a logical and mathematical equivalent of the sentence: "Hereby it is proved that this whole sentence cannot be proved." There is an equivalent of this paradox in computer science concerning the procedure examining whether a given program will stop or will work indefinitely.

We discuss these logical paradoxes together because the essence of each of them is the property of reflexivity. There is always an "object" (a sentence predicating truth or falsity, a class, a proof of truth or falsity of the statement, a procedure examining how a program stops), which enters into a relation (being an element, predicating truth, predicating when something stops) not only with other elements of the same type, but also with itself. One could say that these paradoxes have a similar logical structure, or that they are isomorphic. This isomorphism can be easier illustrated by formulating an antinomy analogous to Russell's antinomy of classes for predicating the truth of sentences (and thus for Gödel's proof and the liar paradox). It goes like this: "Does a statement which predicates of all statements (of a certain type) not predicating of themselves, predicates of itself, or does not predicate of itself". It is easy to see, as in the antinomy of classes, that both answers lead to contradictions. And similarly, as in the case of the antinomy of classes, the only solution seems to be a "theory of sentence types". In other words, a logical sentence could predicate only of a "lower type" sentence, that is, a sentence that is at a lower level of hierarchy. In this case, however, both the liar paradox and Gödel's proof lose not only their validity, but also their sense.

Let us present it in still another way. When we make statements, we implicitly assume that they do not refer to themselves. It is easy to demonstrate, even using the example of the liar paradox, that statements predicating of themselves automatically lead to contradictions. (While the statement "I'm telling the truth" is a tautology, the statement "I'm lying" is self-contradictory.) To avoid contradiction, we must limit ourselves to statements not predicating of themselves. In order to do so, however, we must first determine whether a given statement refers to itself or not. One statement requiring such examination is the statement predicating of all statements not predicating of themselves. Subjecting it to the said test, however, leads automatically to the antinomy isomorphic with Russell's antinomy of classes. Emergence of this contradiction is inevitable. It is a similar situation with the numerical equivalent of Gödel's law. The question isomorphic to the antinomy of classes can be formulated as: "Does the program examining stopability of all programs which do not examine their own stopability, examine its own stopability or not examine its own stopability?" The word "stopability" is undoubtedly somewhat awkward, but allows for a relatively clear and brief formulation of the question. In this case, the program that examines its own stopability also contains an internal antinomy, as the predication (adjudication) of stopability determines by definition termination of the program. Therefore, a program ascertaining its own unstopability would be self-contradictory. On the other hand, to determine their own stopability, it has to examine whether it would stop while examining its own stopability, and this can only be achieved by examining whether it will stop after examination of its own stopability at the lower level of hierarchy, and so on ad infinitum, so it will never stop. Thus, such a program is not able to ascertain its own stopability or unstopability. In order to consider this program as meaningful, we need to determine whether it does not examine its own stopability, and this leads to the alternatives expressed above. Here, too, both answers lead to contradictions, and here also the only solution seems to be a "theory of software types". In this example, however, we can most clearly see that a "theory of (any) types" is only a bypass, and not a solution to the contradictions, as nothing like a hierarchy of procedures exists "naturally" in numerical programming, and recursive procedures, that is, those referring to themselves, are commonly used. It is difficult to forbid a software programmer to use them, as they "exist", work, and are sometimes very useful.

Besides, a theory of types, both in the theory of classes and in mathematics, is intuitively artificial, inelegant and deprived of any justification except for saving the above-mentioned systems from internal contradictions. However, since in accordance with the presented view, internal contradictions of both the whole crystal of concepts, and individual conceptual maps are inevitable, we will abandon the theory of types. In fact, we want to emphasize the fact that the discussed paradoxes are derived from the structure of the conceptual network. Their essence is the property of reflexivity. Contradictions can be either bypassed by adopting some "hierarchy of types”, or regarded as an inherent property of the system. The latter is the main purpose of our analysis of the liar paradox, Russell's antinomy of classes and Gödel's proof. In particular, we want to show that these paradoxes are of fundamental (basic) nature and are closely analogous to the contradiction underlying the lack of legitimacy of the presented system (the property of reflexivity of concepts). 


\section{The Conceptual Network and Mathematics}

On a par with logic, mathematics claims, explicitly or implicitly, to use perfectly, absolutely "sharply” specified concepts. The very syntax of mathematics, its relatively very well-defined set of rules and axioms makes it appear to be a system which is completely autonomous and independent from the world. In such case, starting from its adopted assumptions, mathematics would deliver absolutely valid statements. Arbitrariness and unrestrained freedom to choose its axioms is supposed to make mathematics independent from the world. Thus, the fact of the world's existence or non-existence would be without any significance to mathematics. In such view, the entities defined within mathematics may not necessarily correspond to anything real, and this contempt for the external world is supposed to show its autonomy.

However, the absolutism of mathematical statements is incompatible with the idea of the conceptual network. We have just denied the semantic autonomy of logic, and we remember that since Whitehead and Russell (Whitehead \& Russell, 1910-1913), modern mathematics is based on logical grounds. Speaking more generally, mathematics is also a form of language (and, of course, of the underlying conceptual network). It is a language which, like logic, is much better defined than everyday language. This means that the concepts corresponding to the names of mathematical language are better specified, and the relations between them are more univocal. The differences are so significant that in fact mathematics can be subjectively regarded as an autonomous entity. But it is not. Of course, some mathematical statements can be taken to be absolute "with a sufficiently good approximation". This means that in a relatively large conceptual environment, consistent analysis of such statements does not lead to contradictions. However, this does not change the fact that mathematics can be "absolute" only locally and only approximately. In fact, all the basic concepts of mathematics, such as a point, a straight line, parallelism, or a number come from everyday language. Of course, for the purposes of the mathematical system, they have been better defined or clarified, and their clarification consisted mainly of abstracting from certain accidental features which accompany various designata of these concepts in the "real” world. However, their semantic “core” has been preserved, along with their "basic” connotation relations with other concepts. Thus, in the analysis of the system of mathematics, some internal contradictions are inevitable.

In order to avoid these contradictions, they have been unconsciously pushed down into the layer of axioms (and there was almost unlimited freedom in the selection and construction of these axioms), but in a way not immediately apparent. A total freedom of mathematicians to build their system of assumptions was a cover for this operation. A lot of effort was put into checking whether they are externally (between one another) contradictory, and much less in finding whether they are internally contradictory. Let us take a simple example: defining coordinate axes in space. In mathematics, this is done more or less as follows (speaking, of course, by way of example and simplifying it): we have a continuous, infinite space, we set a point for the centre of our coordinate system, we set mutually perpendicular axes intersecting at this point, and define a standard unit of distance. Our main reservation applies to this last operation. If we once define on the coordinate axis a line segment of the length of 1 , and then we take a line segment twice as long and define it again as that of the length of 1 , then after an analysis we will come to the conclusion that these segments do not differ in anything between each other. Because each line segment consists of an infinite number of points, we can freely stretch and shrink this segment, and it will remain the same segment.

Let us take a vivid example. We will use the surface of the sea as our two-dimensional space. We will define our two coordinate axes with the help of evenly spaced floaters. Now, if water movement disturbs the position of the floaters, some will come closer to each other, and some will drift apart, then within the sea we do not have any measure to detect this fact, as this measure is by definition the distance between the floaters. Thus, if according to some "external" measure we place the floaters on the coordinate axes at every one metre, and after a minute we find that some two adjacent floaters are only 5 centimetres apart, then it is obvious that a whole new coordinate system has emerged. But to detect it, we need an "external” measure, as, by definition, the distance between the floaters is still 1 metre. However, when we define in mathematics a space metric, we do not have anything like an "external" measure. We would need to define it first, and this would move the contradiction one level higher. Thus, the imposition of any metric on a continuous space is an internally contradictory operation. It could be also expressed by the statement that an infinite number of points multiplied by zero sizes of the points will never give any definite real number, but simply indefiniteness. This basic contradiction can be, of course, by some suitable axiological operations, pushed down to a deeper level (e.g., to calculus), where it may be harder to trace, and mathematics unconsciously does so. For this reason, many mathematicians may consider 
this accusation as naive, touching problems that mathematics has long since overcome. However, we argue that this "overcoming" consisted only of placing this contradiction in a network of references (connotations) which was so selected that it became difficult to detect it due to insufficient specification of our conceptual apparatus in this region.

A continuous space can be examined at most by methods of topology, and only a quantized space can have a metric (i.e., coordinate axes with specified distances). Is such a space internally non-contradictory? In the view presented here, a system which would be entirely internally non-contradictory is impossible, but contradictions can appear in a more disguised form. Of course, we do not claim that mathematics is of little value because of its contradictions. Quite the contrary. As any conceptual map, it simply must use concepts that are defined only approximately and locally. In this view, mathematics (along with logic) is the best-defined conceptual map within the crystal of concepts. However, attributing some absolute validity, autonomy, or legitimacy to mathematics is philosophically wrong, and in some extreme cases leads to misunderstandings arising from ignorance of its limitations.

\section{The External and Internal Philosophy of the Conceptual Network}

As we have repeatedly pointed out, in order to create a philosophical system, we have to absolutize a certain concept (or a system of concepts) within the crystal of concepts. So it is with the matter in materialism, the spirit in idealism, the will in Schopenhauer's philosophy, and the concept itself in the presented view. We have shown the benefits of our choice of the subject of "absolutization" above. In short, it seems to allow us to get closest to that unattainable goal of "truth". Of course, not to an absolute truth, as we have repeatedly emphasized, but to that which in our conceptual system we intuitively regard as truth. Until we start to examine a concept too closely, we can tolerably freely use it within our conceptual network. Now, however, we would like to renounce this benefit, at least partly, in order to get more involved in the conceptual network and thus be able to say more about it. However, gaining the quantity, we lose somewhat the quality, i.e., we move away from the top in our cone model. But as long as we remember this reservation, such operation is justified. Its advantage is that because of the operation, we will be able to describe the "world" with a language closer to the conceptual map of everyday language. The disadvantage of this approach is a description which is relatively further from "truth" in our version of "relative logic". We will assume that consciousness is the "absolute entity", whereas the conceptual network is its basis and the area where it operates, as well as its content at the same time. In other words, we postulate "thinking in concepts" in analogy with Wittgenstein's "thinking in language". We absolutize also the "external world". In this view, the conceptual network is a link between sensations coming from this world and consciousness "per se". Of course, as it follows from our original, more general approach, a consistent analysis of consciousness and the world beyond our conceptual network, leads to nonsense, although that can be said about any concept. Absolutization is based on the (implicit) assumption that this kind of analysis is prohibited. We will call such philosophy the internal philosophy of the conceptual network, as in contrast to the more general external philosophy that we have been dealing with so far, it is "internal" in relation to consciousness. When we absolutize the real world, we also assume the material basis of consciousness. Therefore, we assume that it is a derivative of the biochemical and neurophysiological phenomena. In this view, mental processes could be reduced to the electrical activity of neurons, molecularly encoded memory and perhaps some other phenomena which are as yet unknown to us. We will try to demonstrate that consciousness constructed this way must also use the system of connotational concepts, i.e., there is no conceivable mechanism by which concepts (or other "mental entities") could correspond to the objects of the "real world" on the basis of denotation.

The sensations being received by sense organs are just a bunch of disordered signals which appear to be complete nonsense if we do not apply an appropriate "key" or interpreting "algorithm" to them. The vast majority of animals have many, often all such algorithms for decoding sensory stimuli genetically built-in. In humans, these genetic predispositions boil down to only a semantic "nucleus", a "crystallizing centre" of meanings, to several "semantic axes", which later are expanded during accumulation of experiences. A "secondary denotation", assigning concepts to the phenomena of the external world, is created through induction. If a given arrangement of stimuli repeatedly causes the same effect, entering into the same interactions with the already existing conceptual network, then it will be built into it as a new concept. Let us trace how the conceptual network of a newborn baby develops. The little baby has the simplest "axes of meaning", such as fullness-hunger, warm—cold, etc., genetically "implanted". These meanings have a purely biological value. If now, through induction (repeated 
coincidence of stimuli), the mother's voice, smell, and touch become associated with warmth and satisfaction of hunger, then this combination of auditory, olfactory, gustatory (milk), and tactile stimuli becomes the nucleus of the concept of "mother". As the baby accumulates more experiences, this concept will be enriched by new elements, supplemented by visual stimuli, related to other concepts, will be further specified. Let us note the following fact: a baby's concept of "mother" differs significantly from "the same" concept of "mother" in a human adult. The "crystallization centre" of first concepts is built upon basic drives: the drive to satisfy hunger, the safety drive, and the cognitive drive. The latter makes the nucleus of the concept of "toy" from an object given for manipulation. An infant's conceptual network is very poor-these few concepts fill its entire world. At the very beginning, there are perhaps only two concepts—let us call them in great simplification: "mother" and "the lack of mother". Later, as the baby receives more and more stimuli, these concepts start to diversify into many derivatives, e.g., "mother” is divided into "mother proper", "nanny", "father", etc. It sounds somewhat grotesque, as the use of linguistic names for these barely formed concepts is not really legitimate. For, as we have earlier pointed out, normally the names of language correspond to the best specified concepts, those with the highest intensity of the "semantic field". The development of meanings in a child's mind was described by Piaget (Piaget, 1955).

Moreover, when we discuss awareness of a newborn baby, we go introspectively back to the times when we did not have language (it is to some extent obvious, as language is also a form of the conceptual network, and quite complex at that). As the conceptual network develops, new, secondary axes of meanings emerge on the basis of the already existing concepts. Subsequent arrangements of stimuli are placed in the network as new concepts in relation to existing concepts. As we have mentioned above, the concepts corresponding to individual and "real" entities are created by multiple perception of similar combinations of stimuli (sensations). General and abstract concepts emerge (at a higher level, as it were) in a similar way, as a result of the mind's "perception" of many similar complexes of "specific" concepts (both individual and general). Thus, induction is the basic mechanism of forming concepts. In a sense, the great dispute about universals (and the rest of philosophy) can be reduced to neurophysiology. In the development of thinking processes, or the internal, autonomous dynamics of the conceptual network, these processes also generate new concepts through distinguishing them from existing ones. Mastering language enormously accelerates these phenomena. First, language significantly increases the efficiency of using concepts, and second, it allows experience to be gained indirectly, from other persons. The question of whether the development of a newborn's mind looks exactly as it has been described above, is not really essential. It is impossible to describe it exactly, even due to the very weak congruence of the nascent nuclei of concepts with the sphere of language. Thus, our goal is to present the main principle, rather than to precisely report the facts.

This is how we imagine the conceptual network's development through connotations. Its development through denotations is assertorically impossible, because any physical bond between a material phenomenon and its representation in consciousness, or rather any mechanism establishing univocality of the representation relation, is inconceivable. An organism does not know, as it cannot know, what combinations of stimuli it will encounter during its life. For signals from the environment to appear in consciousness as senses, it is necessary to have a key to interpret them. Such a key, at least in humans, is not innate. As we have mentioned, it is formed on the basis of induction, through the repeated perception of similar combinations of stimuli. For a designatum of a concept to be tied with it "in advance" by the denotation relation, there would have to be encoded concepts in the genotype for all the phenomena which a human being could ever think or experience, and learning would simply consist of their selection. Obviously, this is absurd. Here, early Wittgenstein's system also completely fails, as it is based on names - designata of material facts. We should assume that learning about the physiological basis of thinking, i.e., the neuronal circuits responsible for it, would greatly contribute to our knowledge about the structure of the conceptual network. Nevertheless, what we now know about human brain functioning strongly weighs against the originally designational correspondence of concepts (or names of language) and "material facts". Computer models of memory reflecting biological systems and taking into account our current neurophysiological knowledge are based on induction. Therefore, within the internal philosophy, we believe that the theory of connotational meaning of concepts is much better documented than the theory of meaning through denotation.

The intended plan of this work was to develop it in an analogous way to the described development of the conceptual network of a newborn baby. Thus, we do not describe the proposed system from A to Z (this way only a linear "object" could be described, and not a complex network of interrelations). First, we tried to present 
its most general elements and properties, and then to analyse them, to "diversify" them into more specific clarifications, and thus to increase the "resolution" of the presented image. In other words, first we treated it cursorily "all at once", and then we showed the main aspects of the system in a little more detail, to eventually deal more closely with certain selected issues which are less directly relevant to the whole. The basic problem in the implementation of this approach is the fact that in the presented system everything is related to everything else, and when we follow one line of reasoning, we have to abandon hundreds of others. Therefore, undoubtedly, we did not manage to be entirely consistent in that intention, although the overall structure of the work probably reflects the adopted modus operandi. This is the only way to describe a thing that is significantly new as a whole. The present work differs, of course, in its "dynamics" from the newborn baby's consciousness with one important feature-it does not start from a semantic void (if the innate axes of meaning can be regarded as the semantic void).

Let us pose one more question: what is the basis of consciousness? That is, what is the functional difference between the neural networks of human and animals? (If we believe that all animals are deprived of even the nuclei of consciousness, which is problematic; in any case, the level of development of human consciousness is undoubtedly incomparable to any animal species living today). We believe that the essence of the matter lies in the relation of reflexivity. The animal brain is a machine "converting" stimuli received by sense organs into its behaviour (we say it somewhat figuratively, as we are not believers in behaviourism in its extreme form). The system's inputs are directed to the external world (including the receptors within the body, e.g., in muscles or viscera). In humans, a part of this "cognitive apparatus" was redirected to the neural network itself. In other words, the human brain, apart from perceiving stimuli from the environment, began to receive stimuli from itself, and probably did so by using some brain areas which were previously responsible for the association of external signals. Thus, consciousness is based on self-recognition of some mental (neurophysiological) processes within the brain. Its essence is, therefore, the property of reflexivity. If we assume that some rather unspecified mental process is a unit of mind, then this process may be responsible for the perception or association of certain stimuli from the environment. A mental entity would be conscious if the complex of mental processes as a whole was directed not only to the perception of external stimuli, but also to the mental processes it consists of. Thus, it would be an entity somehow isomorphic with the conceptual network, where concepts refer not only to the whole world of phenomena accessible to us, but also to themselves.

Consciousness probably emerged during evolution in a more or less gradual way, so there is a smooth transition between consciousness and its absence. As a matter of fact, we don't really know whether e.g., apes or dolphins that are alive today, have some beginnings of so defined consciousness. It seems likely that such beginnings are present in them and that the development of consciousness in them at a low level was impeded by the lack of a more complex language for communication (as well as the lack of such an excellent manipulating tool as the prehensile hand in humans, which enables implementation of more complex brain commands). Language, being itself a form of the conceptual network, fundamentally influences the possibility of its development. In other words, the creation by human ancestors of natural language within the conceptual network acted as positive feedback, causing a snowball growth of the network, including its elements directed at self-recognition (let us remember that we discuss this problem from the level of the internal philosophy). As we have already mentioned, language greatly facilitates using concepts within the conceptual network. Perhaps this is why we have the introspective impression of "thinking in language". However, the existence of mental objects which do not have any linguistic names strongly contradicts the idea of "thinking in language”, including Wittgenstein's philosophy.

From the above discussion we can draw a general conclusion that the structure of the conceptual network does not change during transition from the external to the internal philosophy. In other words, the absolutization of both poles of the axis "consciousness-the real world" within the conceptual network does not change the properties of the network. This is by no means a desertion from the position of the external philosophy. It can be argued that the external philosophy is a dead end, which consciousness entered as a result of the specific formation of the conceptual network in the real world, and so that the laws governing matter led to such formation of consciousness that proving the absolute existence of the material world within it became impossible, despite the fact that this world does exist. In other words, the evolutionary development of the conceptual network was aimed at the survival of the species, and not at its later predispositions to do philosophy. This is certainly a tempting speculation, especially for materialists and advocates of common sense reasoning. It is hard not be fascinated by this idea of the "consciousness trap". It would explain why, despite the "objective" existence of the material 
world, we are not able to prove this existence. This is simply because "really" existing matter created-within itself-organisms with such neurophysiology, being the basis of the mind, that creating mental images of real objects is not possible through direct denotation, but only through connotation (besides, we have shown that a different mechanism of this assignment is assertorically inconceivable). Therefore, although we live in the "real" world, we are never able to prove its "reality".

Let us present the presumed sequence of events more precisely. On a material basis, some living organisms composed of cells emerge. A certain type of these cells specializes in perception and processing of external stimuli. These are nerve cells (or neurons), and they form a network called a nervous system. During evolution, the nervous system gains the capacity—apart from simple association of external stimuli—for certain autonomous activity (independent generation of stimuli). The flow of information within the neuronal network consists of transmission of nerve impulses from one cell to another. Nerve cells communicate with one another using projections called dendrites and axons, connected by synapses. As a result of experience, a nerve cell acquires memory, and remembers which combination of received stimuli is supposed to make it stimulate another specific nerve cell, and which is not. This memory of the cell has only local significance. This means that it makes sense only in conjunction with the information of other cells it contacts and in what way (and therefore also with the information in other cells; this information in these other cells, in turn, refers to information in still other cells, and so on; eventually, we come to the conclusion that the information in a given cell has meaning only in reference to the information contained in the entire system). Information (memory) contained in a cell that was removed from the nervous system is completely meaningless (as the information about the cell's connections, or its "semantic context", is automatically lost).

Let us show this using a very simple example. Let us take an organism only consisting of several cells and comprising only one nerve cell (we are afraid that such an organism does not exist in reality, but it is not important for our reasoning). This is an aquatic organism, which can feed on organic matter, but in its absence the organism is capable of photosynthetic nutrition. For photosynthesis, light is needed. Our organism contains two receptors which are connected to a single nerve cell: receptor $\mathrm{A}$, sensitive to the presence of organic substances in water, and receptor B, sensitive to light. The nerve cell can stimulate effector C (e.g., a flagellum) which makes the whole organism move in the direction of the light. The cell transmits a signal to effector $\mathrm{C}$ only when it gets an impulse from receptor B (light is present) and there is no impulse from receptor A (a lack of organic substance). Thus, we get a logic function: if B and not-A, then C. Now let us remove this cell from the system. It is still able to perform its logic function, but without the assignment of suitable meanings to $\mathrm{A}, \mathrm{B}$, and $\mathrm{C}$, this function is meaningless. We could substitute A, for example, with the presence of gold in the environment, Bwith the presence of a predator, and C-going in its direction. In such a situation, our miserable creature in the absence of gold in water (which is rather typical) would swim into the mouth of the first encountered predator. It is difficult to regard such behaviour as evolutionary purposeful. Thus, we can see that the sense of information contained in the nerve cell depends on connections with other nerve cells, receptors, and effectors, that is, on the "context of meanings" of the cell. A direct reflection of this fact is dependence of a concept's meaning on the conceptual context in which the concept is located, if we assume the neural basis of consciousness.

Let us analyse the same issue in the case of a more complex neural network - the human brain. As we have already mentioned, the functional unit of this neural network is a nerve cell (neuron). The activity of such a cell consists of the perception of stimuli from other neurons via projections called dendrites, the processing of these stimuli with the participation of the information already possessed by the cell (its memory), and the possible transfer of the processed impulse to other cells with the help of a projection called a neurite (axon). An axon of one neuron connects with dendrites of other neurons with the help of synapses. It is obvious that different neurons, fulfilling different roles, being part of different areas in the brain, must differ from one another. So what do these (functional) differences consist of? We can distinguish two separate categories of these differences.

The first category comprises internal differences. Each nerve cell has its characteristic logic function which transforms the received stimuli into the stimuli sent forward. To put it more simply, different cells make different use of sets of impulses they receive. Some cells reinforce a given combination of signals, sending a strong output signal, other cells respond to the same input combination of signals by not sending forward any impulse. By input combination we mean which dendrites are activated and in which time sequence, whereas by output response-whether and how (we mean intensity and time modulation here) the impulse will be sent further by the axon. The way of assigning an output signal to each configuration of input signals determines the (local) specificity of a given cell. We will discuss the idea of this peculiar processing, or transformation of stimuli by a 
nerve cell in the brain using a very simple example, analogous to the earlier example of an aquatic organism containing only one nerve cell. Let us suppose that in a simple case, a nerve cell has only two inputs (dendrites) $\mathrm{A}$ and $\mathrm{B}$, one output (axon) C, and that these inputs and outputs can be in only two states: 1 (signal present) and 0 (the lack of signal). It should be noted that the identification of dendrites and axons with inputs and outputs is a simplification; we should rather talk about synapses located on the dendrites and the axon. Our cell can perform one of many logic functions. Let it be the function already known to us: if not-A and B, then C; in other words, the output signal C (by the axon) is sent only if the dendrite (input) B is stimulated, and the dendrite (input) A is not stimulated. Another nerve cell can perform a different logic function, e.g.: if A or B, then C. Of course, as the number of inputs (dendrites and the synapses located on them) and the variety of their states (kinds of activation) increases, the number of possible logic functions, transforming input signals into output signals, grows immensely. The kind of function performed can change under the influence of past experiences (activations). We call it memory. The concept of memory applies to both single neurons, and their assemblies. Its physiological (perhaps biochemical) basis is not yet well-known.

Does the logic function performed by a given nerve cell unambiguously define its role and meaning within the neural network? Does it completely determine its specificity? And if not, is this feature a deciding factor at least? The answer to both questions is no. The "meaning" of a given neuron (or a group of neurons) in the functional "context" of the neural network and the entire brain is primarily determined by its connections via dendrites and axons to other neurons and their groups. The function of these "neighbouring" neurons, in turn, is determined by their own "context of meanings", and so on, until the examined network of connections and relations embraces the whole nervous system with all its receptors and effectors. It is only this system that determines, ultimately, the adequate functional context, it constitutes a reference system. Any change of this context modifies the functional identity of a given cell. It should be emphasized that there may be many nerve cells which perform a specific logic function; probably there are many. These cells may serve different roles in different areas; they may participate in various processes and create (along with other cells) the basis for various mental phenomena. However, in the whole brain there is (probably) just one neuron that connects with exactly these and not other cells (groups of cells) in exactly that and not another (functional) way.

Thus, we see that the internal differences between cells (in terms of logic functions performed by them) are quite insufficient to determine their specific and individual roles in the brain. In this respect, the second category of differences is more important, namely, the external differences concerning the environment of a given neuron in the neural network, its semantic context, that is, which other neurons it is connected with and how. The logic function of such a neuron, removed from its environment, loses its meaning, it becomes an empty structure, which, being able to apply to any real content, applies to none. Thus, the information "contained" in a given nerve cell is the sum of the information about its internal "logic structure" and the information about its functional "location" within the neural network (and thus de facto about the structure of the neural network). The first is related to static and local memory, probably on a biochemical basis; the second-with global memory, which may be partly dynamic and consists of the circulation of certain signals in the brain circuits. Only the first type of information and memory can be preserved after destruction of the neural network, and therefore possibly encoded and transmitted genetically. Therefore, only the structure of individual neurons and relatively simple neural networks can be inherited. Thus, the structure of more complex networks, above all those related to the human mind, must evolve during ontogenetic development through acquisition of new experiences. A comparison of the conceptual network structure and the neural network structure leads to one important conclusion. In both cases, the meaning of an element of the network (a concept, a neuron, or a group of neurons) is constituted by connotation, that is, by reference to other elements. The most general structure of both networks is therefore analogous. It is understandable, as, within the internal philosophy, the conceptual network is an "effect" of the neural network activity. However, this fact greatly reinforces the earlier made assumption of connotational structure of meanings in our mind. While we find general similarity between the neural network and the conceptual network, we should avoid, however, too far-reaching analogies. In particular, it would be a mistake to suppose that one nerve cell (or even their simple group) corresponds to one concept. A concept is probably a derivative of a complex functional structure of the neural network, which may not be possible to locate spatially within the brain (such as the olfactory or auditory centres); it evolves over time, penetrating structures which "carry" other concepts.

The discussed above genesis of consciousness (mind) determines its helplessness in the sphere of philosophy, its inability to prove the objective existence of the material world, where this consciousness exists, after all. This 
approach might seem more attractive than the original external philosophy, because it leaves consciousness and the material world "intact". Moreover, it explains why, despite their "real" existence, it is not possible to prove this existence in an absolute way, with which philosophy has struggled over the centuries. However, there are two reasons why this approach is less legitimate than the external philosophy from the perspective of orthodox ontology. First, the internal philosophy is excessive in relation to the external philosophy-it brings into being entities which are already not needed to grasp all the phenomena accessible to us, as it is satisfactorily done by the conceptual network. Therefore, it does not meet the requirement of Occam's razor. Second, as we have already stated, it is impossible to go beyond the conceptual network. Thus, talking about absolute entities beyond its limits is meaningless, as these are simply empty names. An analogous situation is in physics, where talking about speeds greater than the speed of light beyond the horizon of events, or about universes existing in other dimensions and having no contact with our Universe, is considered unacceptable, or at least futile. The theory of the internal conceptual network is undoubtedly dearer to us in terms of its pragmatic value and common sense, but it cannot withstand criticism as a consistent ontological system. Nevertheless, it seems to be useful for practical purposes and provides "locally" good approximation of the external philosophy.

The above criticism of the internal philosophy has been made from the absolutist perspective. However, as we have already said many times, demanding absolute truths is utopian. On the other hand, we have assumed that in our system the measure of truth is the degree of a concept's specification and its coherence with a given conceptual context. It is difficult to find concepts in our crystal of concepts which would be more involved in relations with other concepts, more obvious and fundamental, than consciousness and the external world. The entire structure of the conceptual network is such as if this network "really" was the basis of consciousness functioning in the real world. This is the best proof of the existence of the external world that we can afford. But this proof is perfectly sufficient. If we assume that the question of whether the external world exists is sensible, then the answer, of course, is yes. Whether we consider the question to be sensible depends on how far we are from the apex in our cone model of philosophy, or which of the distinguished levels we are on. The question of the existence of the external world becomes meaningful right after we leave the second level and start going towards the third level. That is why science, logic, and mathematics can act sensibly based on the real external world. Thus, if we can say anything sensible at all, then it is the statement of the existence of the external world. A category difference between mental objects, being elements of consciousness, and material objects, received as sensations, is perhaps the sharpest semantic boundary we can draw at all. The existence of the external world is the only rational explanation for the observed structure of the crystal of concepts. This is certainly not an absolute proof, but such proof is impossible in principle.

If we assume (as we do in the internal philosophy) that the human mind, through induction, creates certain images of objects in the "real world", that is, the concepts being to these objects in the apparent denotation relation, then there is a problem with what these objects "really" are. In other words, we deal here with the "things in themselves" (noumens), about which, according to Kant, nothing can be said (Kant, 1999). However, we believe that this extreme cognitive pessimism is not entirely justified.

Sensations that come to us from things in themselves certainly possess some structure (here we mean not the kind of structure, but the degree of its complexity, measured by information contained in it). Our view of it, the interpretation of this structure, our division of visual sensations into categories, our taking them in terms of time, space, colour, size, and shape, is the property of the human mind. However, if these sensations have some structure after their interpretation by our cognitive apparatus, the mind, then they also had to have some structure before this processing. Information does not emerge out of nowhere. During the transfer of information its amount (according to the theory of information) can only decrease or remain unchanged; it never increases. Processing of external stimuli by our mind is a kind of transmission, the transformation of information. Thus, the structure of stimuli before their processing must be at least equally complex (although the kind of this complexity remains a mystery to us), as sensations that have been already "decoded" by our mind. As a matter of fact, because perception and processing leads to significant losses in the initial information, the complexity of the structure of stimuli coming from the "world in itself" must be much greater than the complexity of the structure of our sensations.

Let us consider this using the following example. If we look at the Moon with the naked eye, then the image that reaches our consciousness is much poorer than the complex of stimuli "really" sent by the Moon. Our eyes have limited resolution which does not allow us to notice details we could see through a telescope. Also, we do not perceive, for example, the ultraviolet radiation, which we can detect with a suitable detector, but we cannot 
see directly due to the range of electromagnetic wavelength to which our visual organ is sensitive. We could give many such examples. These are stimuli which we do not receive directly, but which we can detect using a suitable apparatus and the essence of which we can grasp, to some extent, with our conceptual network, because of the knowledge we have. But there are also some conceivable stimuli that we could neither detect using equipment accessible to us, nor grasp within the system of meanings formed in our minds. After all, we design our research instruments on the basis of what we know; we cannot rule out the existence of something that we do not even suspect could exist due to the lack of appropriate concepts. Loss of information in the process of perception is, therefore, inevitable in principle.

But, is not the above-stated at odds with our everyday experience? After all, if we look at a given specific tree, then we can "see" much more than is contained in our sensations. We can "see" a representative of a certain species of plant, an object containing cells, vascular bundles, etc. That is true, but this "surplus information" does not appear out of nowhere, it is the result of our previous direct or indirect observation (of other trees, microscopic preparations, information in books). The same applies to the "information" contained in dream visions or hallucinations. Thus, the information, the structure of impressions in our mind does not come from nowhere; it is a reflection of some (unknown to us) structure of the "things in themselves". If two sets of sensations appear to us as trees, and two others as elephants, it means that the "things in themselves", underlying the sensations of each pair, have a similar structure, and the structure of "things in themselves" in different pairs is substantially different. To sum up, the (undeniable) existence of the structure of the world within our mind implies the existence of at least the equally complex structure of the "things in themselves".

Therefore, the perception of the world through concepts is most certainly fragmented. We can see only some aspects of the things in themselves. Let us take, for example, an electron. Depending on the method of observation, it appears to us as a particle or a wave. The question of whether the electron "really" belongs to one of these categories is meaningless. In addition, the claim of an electron's "wave-particle duality" is only a simplification created by our mind. The particle or wave nature of the electron is most probably a manifestation of a much richer structure, irreducible directly to a particle or a wave, being something more than their "sum". Let us use the analogy of an egg. When we look at it from above, from the side of its tip, it appears to us as a circle, and if we look at it from its side, we see it as an egg-shaped ellipse. Let us suppose that we are not able to see the egg in three dimensions, but we have access only to its projections on two mutually perpendicular planes. Then also, depending on which plane we look at it, we see it as a circle or an (egg-shaped) ellipse. But does this show the "duality" (double nature) of eggs? As this egg is something more than the sum of a circle and an ellipse, so an electron probably has a much richer structure than we might think on the basis of simply adding its particle and wave properties. The situation of an electron is quite unusual. Most "things in themselves" appear to us only in one aspect, thus it is much harder for us to realize its impoverishment in relation to the "original".

\section{The Conceptual Network and Science}

In Section 2, we have already dealt with many aspects of mutual relations between the crystal of concepts in general and the map of science in particular. This was undoubtedly a breach in the declared plan of this work, in which we wanted to move gradually from the most general statements to details, clarifying various aspects of the main idea as we go. However, we can justify this deviation by pointing out that we have used the structure of the conceptual network of natural sciences as an example of the construction of the conceptual network in general. We used the map of science for this purpose as it is well-separated (distinguished) from other maps, and because the maps of its particular disciplines are relatively well-isolated, largely due to historical reasons. Thus, the properties of the conceptual map of science have been earlier characterized.

In this section, we will deal mainly with the impact of science on the development of the conceptual network. Or rather, as science itself is also a part of this network, we will discuss its impact on philosophy. As we said earlier, philosophy is kind of a buffer zone, separating areas of the crystal of concepts characterized by high intensity of the field of meanings from the semantic void. Therefore, the development of philosophy to some degree defines (or rather reflects) the evolution of the whole crystal of concepts. Thus, the phrase "the impact of science on the conceptual network" is not significantly different from the phrase "the impact of science on philosophy”. The very fact of putting this problem in a separate section suggests that we assign a special place for science in the conceptual network.

And indeed, as language is a universal tool that greatly increases our ability to use concepts (and thus the de- 
velopment of the conceptual network), so science is currently the main generator of new concepts having a significant relation with reality. This should be understood as follows. New concepts emerge as a result of separation from the existing concepts through creation of new semantic relations between them, so far non-existent. The "parent" concepts are also modified as a result of interactions with newly formed concepts-they become better specified. The process of creating new concepts is associated with "diversifying" meanings on the semantic axes. Of course, new concepts emerge on the whole area of the crystal of concepts. This process is especially efficient and the emerging concepts are particularly well-defined in places where the conceptual network is matched by a rich and structurally varied language. Language plays a very important role in the process of generating concepts, but it is rather a "channelling device", and therefore a passive factor. However, we still need an active factor, that is, a generator of concepts. We will distinguish two such generators: primary and secondary. The primary generator is the "internal activity of the conceptual network", that is, the processes of thinking.

At this point we need to make a digression, which actually should have been made earlier. Now, the entire discussion contained in this section makes sense only within the internal philosophy, that is, when the conceptual network is treated as a basis of mind (without being identical to it) and as this mind's link to the "external world". The external philosophy treats all regions of the crystal of concepts (including the mind and the real world) as equally legitimate. In accordance with the previously quoted rule, it is closer to "truth", but we can say less within it. If we want to discuss the aspects of the world which are interesting to us, we have to choose the middle ground between the opposing poles: the silence of "absolute truth" and the complete arbitrariness of statements. The more legitimate (in an "absolute" way) is supposed to be what we say, the less we can say. At the end of this digression we should note that it reflects the reservation made above that the truth (or sense) of statements is relative to the level (or the conceptual context) at which they are made.

The primary generator of concepts - the mind and thinking — can sometimes work very efficiently, as long as it operates with a well-defined conceptual map, or, what is equivalent, a well-defined layer of language. Mathematics is the best example. In mathematics, there are highly refined concepts accompanied by language with a rich and ordered structure. This allows verification of statements, and therefore the avoidance of arbitrariness, which greatly helps to specify newly formed concepts (by verification we should understand a process which ensures that a given conceptual system is internally consistent "with good approximation"). In other words, the conceptual network is so well-specified locally, that the newly formed concepts are also well-specified. However, this verification is somehow internal in relation to the conceptual network (let us remember that we operate within the internal philosophy).

The external verification of concepts can be ensured only by the external world. The confrontation of concepts with the world constitutes their secondary generator, and that is why we should assign such an important role to natural sciences (perhaps the process is not "generating" in a strict sense, but at the level of the conceptual network such semantic distinctions are fading). Here, verification is much stricter than in the conceptual network itself. This is due to the lack of relativization by connotational relations; in the internal philosophy the external world is "absolute" and "in itself". Even the exactness of mathematics is not completely autonomous, as it uses objects (such as a number, a point, a straight line) derived originally from the world (or its projections on the everyday conceptual map). Thus, while the relation of strict denotation between the conceptual network and the real world is impossible, the facts of this world constitute points around which the conceptual network must tighten, if it is to keep contact with the world. And because this just what the pragmatism of biological evolution requires, our conceptual network is somehow adapted to exploration of the external world. On the other hand, what was sufficient for our ancestors to hunt mammoths, now could fail in further exploration of the world, when natural selection has practically ceased to work. In other words, the structure of the conceptual apparatus is definitely not pre-adapted by evolution to solve scientific or philosophical problems.

Moreover, our mind is directed not only by the cognitive drive, but also by other drives (e.g., self-preservation instinct, and generally - the drive to stimulate the reward system in the brain) pulling us away from the "objective truth" in the direction of mental comfort. However, the principal obstacle to gaining the "absolute" knowledge of the world is the connotationality of the conceptual network. On the other hand, the connotational mind is the only mind possible, and as such it must function with contradictions and inconsistencies. An entity somehow denotational, e.g., a computer, will remain in principle non-mental (we could, of course, model a connotational mind on the computer, but then it would have to consist in the repetition of biological evolution; such mind would operate at somewhat higher level than the operating structure of the computer).

We can give yet another example of the necessity of internal contradictions in a mind created by biological 
evolution. Logical analysis will always show that "nothing has any meaning”. In other words, it is impossible to justify the meaning of anything. However, an individual deprived of any sense of meaning of life (motivation, intentionality) is worthless from the perspective of biological evolution and will be eliminated by natural selection. A conscious entity, by the very fact of existing, falls into a logical contradiction. Therefore, it can emerge only in the process of evolution that has some autonomous "aims" (or rather mechanisms) and it can have only a connotational mind. However, the other (the first, actually) side of the coin is the fact that human cognitive apparatus (and thus its conceptual network) is to some extent directed at examining the external world and processing the data coming from it. The conceptual network's good (at least in the sense of pragmatic, instrumental) congruence to the external world determined evolutionary success. This is why we regard natural sciences, which are sophisticated tools for examining this world, as so important in the process of generating concepts.

Things are different in philosophy. If it is to create new concepts only on their own, then this process must be, in principle, not very efficient, and the created concepts are little more meaningful than empty concepts. For philosophy has neither a well-defined conceptual network (and thus well-defined language), nor direct contact with the world. Thus, it has neither the internal, nor external possibility to verify its concepts. Therefore, contemporary philosophy is bound to assimilate concepts from the exact sciences and this is practically the only opportunity for its development. The above sentence expresses the main idea of this section. In other words, the exact sciences should be regarded as the main generator of concepts (and paradigms) for philosophy. Instead of preceding science in the exploration of various aspects of reality, as philosophy has done so far, it must now wait for science to form new concepts and new ways of grasping reality, which it could use. But the relationship between philosophy and science has not been entirely reversed. Philosophy, as a "semantic buffer" on the border of the semantic void, will always in some way precede science in the description of the world. Let us take, as an analogy, the example of biological succession. Just as on a bare rock first lichens appear and change the basis, making it available to other plants, which then gradually supplant them, so philosophy is preparing some semantic spaces (even if only by asking relevant questions), which then are taken up by science, clarifying and verifying concepts provisionally formed by philosophy. Thus, we can speak about mutual stimulation. However, it is almost a truism to say that the centre of gravity has shifted decisively in the direction of science.

This work would have never come into being without deriving from science some concepts, semantic axes or paradigms, such as continuity, field, maps, or the concept of relativity. They come mainly from the sphere of physics and mathematics. However, particular concepts are not as important as the way of seeing the world; and in this respect, science has overtaken philosophy.

The clash between the conceptual network and reality (at the level of the internal philosophy) is a strong generator of new concepts. Incorporating new concepts in relation to the existing ones, the "budding" of new concepts from the existing corpus of the conceptual network, determines the conceptual development of science, and that means the development of its conceptual network. As the conceptual network "gets denser", new answers and new questions emerge, the existence of which cannot even be sensed on the basis of a simpler conceptual network. On the other hand, the concepts which were previously well-established and intuitively perceived as clear and unambiguous, now become "fuzzy". Physics and neurophysiology provide good examples of the processes described above. In physics, the concept of matter which was previously clear and obvious, a perfect Alpha for many people, now practically does not exist. It has been dismantled into the concepts of field intensity, equivalence with energy, particle-wave duality, and probability function. Einstein's general theory of relativity is a great example of the phenomenon of relativism and relational nature of meanings in a conceptual system - the concepts of space, time, matter, gravity, speed, and energy are so formulated there that they explicitly have meanings only in relation to other concepts. If we removed one element, the whole structure would collapse. Thus, for example, the removal of matter would change the structure of space-time dramatically. Similarly, in neurophysiology and neuropsychology, the concept of spirit (consciousness) is gaining attributes that have never been sensed before, and is losing its obviousness and intuitive clarity. Physiological and molecular bases of perception, memory, and association processes, as well as the impact of external factors on the state of consciousness, consistently reduce the area reserved for the "spirit in its immanence", or "pure consciousness", and increasingly indicate the secondary character of consciousness in relation to matter (on the other hand, matter is received by us only through our consciousness). Thus, the intuitively homogeneous picture of spirit as an integral and autonomous entity has been largely ousted, at least in the circles which are willing to acknowledge this state of affairs. And again, the original concept of consciousness is "budding" with derivative concepts, 
which "take with themselves" some of its content, some of its connections with other concepts. Of course, here science has not yet gone as far as in the case of physics. We still cannot break the very core of the mind-the sense of consciousness, or ego-into specific concepts. Will it ever be possible? We believe that it will happen to the extent that it did with matter: the very concept will begin to slip through our fingers, and the name, not designating anything absolute or indivisible anymore, will become empty. One of the attempts to "break down" consciousness into simpler concepts was presented in the previous section.

The differences between the primary generator and the secondary generator of concepts (and thus between the internal activity of the conceptual network and its confrontation with the external world) can be characterized differently, with even bigger emphasis on the role of natural sciences in the development of the conceptual network. Even in the best-specified conceptual maps, such as mathematics, the meaning of concepts is defined only with some approximation, although the "semantic uncertainty" may be very small. Let us imagine that a given conceptual map is expanding and creating new concepts. On the basis of these new concepts, still other concepts develop. In this way, some "conceptual chains" emerge in the semantic space; these are strings of primary and derivative concepts in the conceptual network. Now, in such chains the indeterminacy of meaning of these concepts adds up as we move along a chain. Sufficiently long chains, even those composed of very well-defined concepts, will start to "bend", or "deviate" from their intended line in the semantic space. Susceptibility to these "deviations" is a measure of "semantic uncertainty". Therefore, verification of a system's consistency in the primary generator of concepts is only limited and local. For if some conceptual chains develop independently of each other from a conceptual nucleus, for example, from an everyday conceptual map, then in sufficient distance from this nucleus they start to contradict one another (as an example, we can give various religions and philosophies). Thus, in a global sense, the verification of the conceptual network structure is certainly insufficient in the case of the primary generator.

It is different with the secondary generator. In natural sciences, all newly emerging concepts or larger areas of the conceptual network are confronted with the external world right away (or with some finite delay). For example, the Big Bang theory and the Steady State theory coexisted for some time in the absence of evidence falsifying one of them; they only reflected philosophical inclinations of their advocates. However, the discovery of microwave background radiation (and many other phenomena) settled the issue in favour of the Big Bang theory. Thus, the external objective world in a sense determines the lines in the semantic space, which the conceptual chains must "conform" to. Of course, their mutual congruence (that is, of these lines and chains) is never perfect. However, semantic indeterminacy does not add up as the chain develops, but remains more or less constant. Thus, assuming that the external world is non-contradictory, it imposes its non-contradictoriness (of course, only imperfectly and approximately) on the conceptual network structure. Of course, this applies directly to the conceptual map of science (the everyday conceptual map has long been, to some limited extent, agreed with the external world). Indirectly, however, philosophy and everyday spheres are also under this influence. Thus (we discuss it still from within the internal philosophy), the crystal of concepts not only could not have emerged without the external world, which is a rather trivial statement, but this world is necessary for its development as a whole. And because currently only natural sciences have adequate methodology (experiments, observations) allowing the measurement of the degree of correspondence between the "real" world and the conceptual network, we believe that they are the main opportunity for the development of philosophy.

It is a different matter with broadly understood culture and humanities (and philosophical systems drawing from them). First of all, they lack the external—discussed above—verification of their claims, and their internal verification is very weak. The evolution of natural sciences created in different cultures must converge-its convergence determines the congruence of the structures of these sciences to the structures of the world. The methodology of natural sciences is able to eliminate any deviations from this structure; thus, it operates on the principle of negative feedback. In contrast, the development of culture consists of reinforcing some accidentally chosen direction and further going along this path. Culture determines the course of its further evolution through the already possessed structure. Thus, it operates on the principle of positive feedback: the more it explores a certain path of development, the more it is committed to it. Because the taken direction of development of a culture is accidental, the evolution of various cultures is divergent. When the disciplines of humanities study culture, they simultaneously create it. In natural sciences, a subject of their research exists independently of them. Culture and humanities create the subject of their research themselves. They start from the biological constitution of human beings, but it is not their object of interest - they are interested in what is built on it. Learning new structures is essentially indistinguishable from creating them. This creation has no limits, in the sense that 
the created structures do not have to conform to anything except themselves. There is complete freedom and arbitrariness. Culture needs only to have "some consideration" for the biological constitution of human beings and physical realities of the world. For example, it is limited by the law of gravity or by human mortality (although it is not difficult to find within it systems that even circumvent these basic facts). However, apart from this necessary minimum, it does not have any points of contact with the objective world.

Two independently developing physics (e.g., in two different cultures or on two different planets) might have different starting points (within broader culture) and might use different concepts. However, their structure would have to conform - better or worse - to the structure of the objective world. And as this world, however it is understood, should be regarded as unambiguous, they would also have to conform to each other on the basis of transitivity: if $A$ is congruent to $C$, and $B$ is congruent to $C$, then $A$ is congruent to $B$. Thus, to the question of whether it is possible to have two different physics with two different underlying mathematics, we can answer: yes and no. Yes, because they can start from different concepts taken from everyday language, they can use different starting assumptions, and they can be (to some extent) differently directed by stochastic processes. No, because the structures built within them must conform to the structure of the objective world, and therefore to each other. This protects natural sciences against arbitrariness. Humanities tacitly accept the fact of existence of the objective world, but they focus their attention on human culture that is developing to a large extent in an accidental way. This fact, combined with the lack of verifiability, makes humanities and culture a very uncertain base for philosophy.

\section{The Conceptual Network, Mind, and the External World}

Perhaps the most evident problem of philosophy—and the closest to everyday life—is the dichotomy of spirit and matter. This dichotomy has stimulated the development of philosophy for most of its history. It was done in various ways.

First, in every generation of thinkers there were those in favour of one or the other alternative of the choice "either spirit or matter", and arguing for priority of the chosen one. As the crystal of concepts of humanity developed, particularly the crystal of natural sciences, each group could quote more and better "specified" evidence for their option. The conceptual apparatus of science quite naturally induced embracing materialism, rather than idealism. Nevertheless, nobody has managed to indisputably prove the superiority of either of them.

The second option is dualistic philosophy-the coexistence of spirit and matter as two fundamentally different categories of phenomena. In this situation, however, a mechanism of communication between them remains a mystery. Any deeper analysis of the problem breaks the symmetry and demonstrates priority of either spirit, or matter, depending on the direction of the philosophical argument. Dualistic philosophy is also suspect on account of Occam's razor - two different kinds of being are already quite a lot for this orthodox ontology.

Other philosophers, sceptical about the possibility of making a meaningful choice between these two alternatives, tried to reconcile them, that is, they tried to prove that the opposition between spirit and matter is only apparent, and that in fact these things are manifestations of some sort of unity on a deeper level of cognition. Such a paradigm of solving the problem (incidentally, it was borrowed from science) is undoubtedly intellectually satisfying, but there is the problem of identity of this deeper level. For example, Russell's neutral monism (Russell, 1975), replacing the materialistic or dualistic monism and dualism, boils down to calling everything that exists "Unknown". The problem is that whatever we reduce matter or spirit to, it should be "like something", so that we can associate it with something familiar in our conceptual network. The concepts of the real world and mind are associated in our crystal of concepts with such a huge number of concepts that we perceive them as extremely clear and obvious. To replace them with something that is supposed to be their essence or basis, we need to make this something which is at least comparably obvious and intuitively acceptable. Therefore, it is necessary to link it to a dense network of semantic relations with the "everyday" conceptual map. Thus, it is not enough to say that spirit and matter are one, but it should be also said what they are and this choice should be justified.

Finally, the fourth approach consists of searching for an Alpha different from spirit and matter, and replacing them in their function. Such a role was played by Leibniz's monads or Schopenhauer's concept of will. But no one has managed to persuade humanity to embrace these new Alphas for the reasons mentioned above- they take away concepts of spirit and matter which are very well grounded in our consciousness, and give in exchange something that people do not really know what to do with. The old dichotomy between consciousness 
and the external world is still the basis of philosophy in the popular imagination as well as a certain "yardstick", a reference for newly created philosophical systems. Spirit and matter are such a strong "binder" of the everyday conceptual map that each new philosophy must indicate a place in it which is occupied by these two "categories of phenomena". In other words, an ontology aspiring to describe all that is accessible to us should explain how we should understand the concepts of "spirit" and "matter". Thus, we will now define their place in the presented view. If we were forced to classify it in one of the four above-mentioned approaches to the dichotomy, then certainly it is most similar to the third group of philosophies, although it obviously also shows some parallels with the fourth group. However, some properties place it outside the quoted classification, somewhere near the self-invalidating philosophy of early Wittgenstein. But let us get to the point, that is, to explain what spirit and matter are in our view.

In fact this section could be regarded as unnecessary, as it is easy to describe the place of both above-mentioned Alphas in the presented philosophy on the basis of what has already been stated. But, first, as we have already said, one thing is a bare statement, and another thing the same statement with an accompanying system of clarifying concepts. Thus, reference to spirit and matter will serve to describe the system further. Second, the topic will offer an opportunity for several related digressions that otherwise would remain isolated elements of our discussion. Third, this section will be an opportunity for a certain summary of what we have said so far. Fourth and finally, it will help to locate and refer the presented view to the system of "everyday" concepts which are best intuitively grounded in our mind. Thus, we believe that introduction of this section is justified.

Because spirit and matter are treated in the internal philosophy of the conceptual network as "absolute" Alphas, as in "classic" philosophy, they cannot be analysed within it. Therefore, now we will refer matter and spirit to the external philosophy, making a sort of reverse operation in relation to the one we performed earlier when we went from the external philosophy to the internal philosophy. Within the external philosophy, both of these Alphas are concepts, like any others, or, if we treat them more broadly, they are conceptual maps. Between these two categories there is no absolute boundary, as we have repeatedly emphasized. That is all, if we were to summarize this section in one sentence. However, it is difficult to deny that intuitively, "at first glance", the two mentioned phenomena differ from each other radically_from a philosophical point of view, these are perhaps the two most opposite things one can imagine. And yet, we do not even need to go down to the level of the conceptual network to "agree" them. Of course, it is nothing new to say that the "real" world is not given to us in its immanent form, but only in the form of the experienced sensations. In addition, our own mind is not given to us in a different way than through "experiencing" thoughts. But is not the very relation of experiencing by its essence evidence of some absolute, autonomous existence of the experienced (object) and the experiencing (subject)? No, because the concept of "experiencing", transferred from everyday language, loses some of its attributes here; they are not associated with it in a necessary way. This is yet another example illustrating the claim that the identity of a name does not prove the identity of its conceptual referent, and moreover, that the concept transferred to another region of the conceptual map must, more or less, change its meaning. Coming back to the concept of "being experienced"-in the new conceptual environment it is no different from the concept "exist", whatever it means.

Thus, our mind, including a sense of ego, exists no less and no more than the "real" world. There is no absolute boundary between sensations from the external world and "purely" mental acts (thoughts), which is best exemplified by dreams or feelings after taking psychoactive drugs, such as hallucinogens. Thus, all the phenomena accessible to us appear as some kind of non-hierarchical field of sensations. How is it different from the previously discussed semantic field, or the conceptual network? First, we undoubtedly associate it differently. We intuitively receive the conceptual network as a static entity, while the field of sensations is something taking place over time (in the sense that the sensations "reach" us, and the concepts "are here"; after all, the changeability in time is also a concept). Moreover, it would be difficult to define a determinant, differentiating sensations within the field of sensations, as the intensity of the semantic field and the axes of meaning differentiate concepts within the conceptual network. Finally, the field of sensations does not show, at least not in a clear and direct way, the property of reflexivity, although it can aspire to universality. Nor is it such a useful tool for analysis of the totality of phenomena accessible to us. As for its dynamics (in the sense of "reaching" from somewhere to somewhere else), this is, of course, also a sensation (or a concept) as any other. Thus, if in a sense we could equate the conceptual network with the field of sensations (we are not able to show any absolute category difference between them), the former is more suitable as the basis for a philosophical system. This does not mean, of course, that we implicitly insist that "everything is a concept". We do not specify what the world is, but 
only what can be said about it. In an absolute sense-nothing. However, if the logical value of the uttered sentences in the context specified here is (in our subjective sense) to approximate 1 , rather than 0 , then we can say about the world more or less that which constitutes the content of this work. In the case of such a poorly defined conceptual network, as it is in philosophy, logic loses its validity (and, in fact, even its sense) and must be replaced by a conceptual vision.

But let us go back to spirit and matter, that is, to mind and the external world. Now, the world is no different in any attribute from the concept of "the world", similarly, in the semantic analysis the mind is indistinguishable from the concept of "the mind". We just associate each name in both pairs differently. But, of course, the "identification" of these phenomena which are so different takes place at a level where almost complete desemantization of the crystal of concepts is going on, and thus, close to the first level (of silence). This does not change the fact that on the third level, in a well-specified conceptual network, the meaning of the name "the world" is in complete opposition to the name "the concept of the world". This second relation is neither "more true", nor "less true" than the first one-it simply takes place on another level, in another context. While the first approach is closer to the "Absolute", the second "means more" by being grounded in a much better specified conceptual environment. Thus, we have come to a kind of principle of "the equality of levels" — sentences which are totally contradictory to each other in the layer of language and logic can be equally true, each at a different level, only if they are in the same degree "compatible" with the semantic context of their levels. As we progressively move between the levels, there is also a gradual change of the "logic value" (if this term still has any sense) of a given statement. This means that at the third level, spirit and matter are what we intuitively believe they are, that is, two distinct categories of phenomena. However, when we are doing any semantic analysis of these categories, we inevitably approach the second level, and each analysis of them that is performed consistently to the end results in a complete desemantization (that is, level 1). Thus, when we agree to a logical analysis of any concept, we fall into a trap, a funnel, where we can only move down, and the freedom of manoeuvre decreases with time.

Thus, any analysis should be done only on a small scale, in order to specify concepts and eliminate concepts that have nothing in common with reality. The problem is to know where to stop, in order not to be left with an empty linguistic name that no longer corresponds to any meaning. The destructive power of logical analysis is visible in the fact that using it, we can demonstrate non-legitimacy (in an absolute sense) of any philosophical system, including the one presented here. Logical analysis is mostly justified between the third and second levels, where we can place science, logic, and mathematics. However, near the second level it loses its legitimacy, as it itself succumbs to desemantization. It is replaced here, as it must be, by a subjective, intuitive sense of truth. The philosophy presented here is undoubtedly logically contradictory or at least non-legitimate. However, first, every conceptual entity is necessarily contradictory, and second, this contradiction is explicitly recognized here and serves as one of the cornerstones of the system.

If we make an Alpha out of spirit or matter, then until we subject them to logical analysis (and this operation is contrary to the essence of the Alpha), we can subsume under it a majority of phenomena accessible to us. However, this autonomy and legitimacy of the Alpha lasts only as long as we provide it with this "immunity". The same applies to other Alphas. But the legitimacy of the Alpha's establishment may not necessarily be totally questioned. As we have shown, no Alpha is able to describe the whole world (the set of accessible phenomena) with satisfactory approximation. However, giving up such universality, we can assume the existence of partial Alphas, corresponding to different aspects of the world. Materialism is a perfect philosophy for natural sciences, idealism—for psychology and religion, and both these paradigms coexist in the everyday conceptual map. The necessity of partial description is a fundamental property of the world in which we live and of the language (the conceptual network) that we use.

Let us illustrate this with a geometric example. Our task will be to approximate a circle with a straight line. It is impossible to do this using a single line-this line will perfectly describe the circle near the point of contact, but as it goes further away the inadequacy of the description will increase to infinity. However, when we have three line segments and make a triangle circumscribed about the circle with them, we can claim that we have created a certain approximation of the circle, although very inaccurately. By increasing the number of segments, and thus by creating polygons with an increasing number of sides circumscribed about the circle, we obviously improve the adequacy of the description. However, we simultaneously decrease its universality, because then we have the world broken down into an enormous amount of conceptual maps. For practical reasons, the best solution would be a golden mean, that is, using a relatively small number of relatively long segments. How, in this situation, are we to describe the operation of science, trying to agree conceptual maps of their sub-disciplines? 
Let us develop further our geometric analogy. Let us suppose that by using suitable energy, we could bend the segments to the circle. In this way, we could also get an equally good adjustment (measured by the distance of the segments' ends to the circle) for a lower number of bent segments or a better adjustment for the same number of them. In the quoted analogy, the energy needed to bend segments (to agree with the conceptual map) corresponds to the complexity of the conceptual apparatus used in order to obtain a good "congruence" of maps. Grand Unification would consist of combining shorter segments into longer ones and "bending" the resulting entity (through increasingly complex conceptual structures) to the circle, that is, by bending a segment so that it is best adjusted to the circle. However, as we would lengthen this unified segment, the energy needed to bend it (that is the level of complexity of the conceptual apparatus) would grow exponentially. We would require infinite energy, that is, an infinitely complex conceptual system to describe a circle with a single, very curved line. We should suppose that this task would soon exceed the capacity of the human mind. Therefore, Grand Unification of "everything that exists" is fiction. It is necessary to compromise. We should be worried that, for example, in physics, we are already close to the threshold of the conceptual system's complexity which we will not be able to cross (now the pace of development and the importance of achievements of theoretical physics has significantly decreased, compared to the beginning of the 20th century). Thus, we will probably have to give up the Grand Unification and set ourselves some closer and less ambitious goals.

\section{A Few Examples of Conceptual Network Analysis}

The simplest form of analysis is breaking down a concept "into its most basic constituents", distributing its meaning between the concepts which remain in direct connotation relation with it, and this way- "un-defining" it. After such treatment, the linguistic name corresponding to this concept is an empty name. The act of desemantization of a concept in the process of semantic analysis is a direct derivative of the property of meaning through connotation. Any concept is by definition subject to this process, but, of course, not all of them at once-something must constitute the basis of the analysis apparatus itself, which after all also consists of concepts. An example of bringing the conceptual analysis to the stage where there is only an empty name left is the analysis of the concept of "cause" performed by Hume. He stated that we can only observe some coincidences between certain preceding events and certain following events in time and space. There is no place for an efficient cause between these events. In a sense, this reasoning is a special case of the method of desemantization of general concepts through indicating the lack of absolute justification of induction. Another example can be provided by the analysis of the concept of "justice", mentioned earlier. If you consider this concept instrumentally, then it boils down to the concepts of "prevention" and "retribution" (understood as revenge). This means that these two concepts are sufficient to explain and motivate all actions undertaken within the administration of justice in the broadest sense. In spite of this, we intuitively feel that there is also something like an idea of a sense of justice. Here, however, it dissipates in the conceptual void. Of course, this does not mean that a sense of justice "does not exist" (after all, we feel it in our consciousness), but only that applying the apparatus of logical analysis to the concept of "justice" strips it of some elements, in fact making it a different concept. A similar process occurs in Wittgenstein's programme of the "clarification of sentences" as the superior objective of the whole philosophy. Such "clarification" leads, on a small scale, to better specification, but also to the impoverishment of the concepts corresponding to the names of language, and when it is performed consistently to the end, it completely desemantizes language. The idea of "absolutely clear" sentences is—in this view—an obvious utopia. The sentences can be clear in our introspective feeling only until we look at them more closely and start to analyse them. Without the principle saying that "perfectly clear and obvious is that which we are not dealing with at the moment", it would be difficult to use the crystal of concepts at all.

It is known that physics is not yet a completed science and that it still has many gaps and contradictions. Let us suppose that such gaps and contradictions concern an elementary particle called "electron" (which is a fact, actually) or, more broadly, that the concept of the electron is not fully defined within the conceptual map of physics (which must be a fact). If now a chemist, trying to infer the properties of chemical compounds from the description of electron orbitals in the framework of quantum mechanics, wanted to keep this constantly in mind, he or she would never carry out their intentions, forced to use a concept which is not entirely and absolutely specified (defined). They must "put aside" the concept of the electron to the periphery of their attention as absolutely clear and specified, temporarily take it at face value as valid in an absolute way, although, of course, it is not. The whole crystal of concepts works on this principle. We cannot focus our attention on the whole area of 
the conceptual network all at once. At a given moment, we are able to deal with only a small region of it, whereas the whole rest of it, being at that moment on the edge of consciousness, is beyond the reach of the apparatus of logical analysis, and that is why it seems absolutely valid and specified. Of course, when we move our attention to another point of the conceptual network, this point, in turn, becomes susceptible to analysis, and the previous object of our analysis will seem absolute to us.

If we were able to grasp the whole crystal of concepts all at once, it would lead to its immediate desemantization. Of course, we can carry such considerations only within the internal philosophy, where consciousness achieves autonomy in relation to the rest of the conceptual network. The described mechanism explains why the concepts usually appear to us as absolutely valid. We simply believe that we operate on the basis of a perfectly legitimate system of concepts. We think that we may correct its small fragments and thus eliminate certain gaps and contradictions. We assume that this way we can achieve perfect clarity and specification of the system of concepts. This assumption is evidently incorrect, as any change of any part of the crystal of concepts affects in some way its whole. The structure of the conceptual network somewhat resembles foam composed of soap bubbles. A new bubble (concept) is budding among other bubbles, pushing them apart, whereas the place of a bubble that bursts is immediately taken up by adjacent bubbles (logical analysis). The tension caused by any change in the system of bubbles is immediately transferred even to very remote regions of the foam. Of course, a certain limitation of this analogy is created by a huge, although not specified, number of dimensions in the semantic space, so that the number of bubbles adjacent to a given bubble is unimaginably large. In a sense, each bubble is (more or less) adjacent to every other bubble. Moreover, the bubbles are discrete entities in contrast to the continuous semantic field. Logical analysis leads to the bursting of bubbles, or perhaps to some merging of bubbles. The bursting of a single, large, ultimate bubble leads to the first level—desemantization of the whole crystal of concepts in an absolute sense.

Another example of an operation performed on the conceptual network is transferring a concept to a different semantic environment. Each concept has meaning in relation to other concepts, through connotation. When we move a concept, we have to break some of these connotational ties, whereas in its new place the concept will be enriched by some previously non-existent links. As the concept's identity is derived exclusively from relations with other concepts, after such transfer we, therefore, deal with a different concept. This leads to an important conclusion: in principle we can transfer not concepts but their corresponding names of language. The same name can designate two (or more) concepts in different regions of the conceptual network. Thus, when we use language, we should be careful not to identify different concepts only because they have the same name, as this can lead to misunderstandings and paradoxes. The best example is the question of whether mathematics is invented or discovered (Barrow, 1992). Serious thinkers advocated each of these options. But this disjunction is only apparent. Both concepts, that is, "invented" and "discovered", were taken from everyday language. We perfectly understand what it means to invent a machine or discover a new land. But it is different with mathematics. These concepts in relation to mathematics mean something different than in everyday language, as they relate to a completely different category of objects. Whereas we usually associate the word "discover" with the statement "to see something that no one has ever seen", and "invent" with "to call into existence a new material entity", in mathematics nothing can be seen and nothing "really" exists, that is, none of them has an attribute that would not be possessed by the other. The only exception is their names. The concepts themselves, moved into the area of mathematics, change so much that they start to mean something slightly different than their precursors. We can say even more-both these concepts are semantically indistinguishable in their new environment.

Let us examine the said disjunction from the perspective of the philosophy of the conceptual network. The emergence of a new mathematical entity (no matter whether "discovered" or "invented”) equals a new concept being distinguished. A certain relation between earlier existing concepts is semantically "reinforced”, and there emerges an area of the semantic field with relatively high intensity and autonomy. The fact that not every combination of earlier existing concepts leads to the emergence of a new concept comes from the fact that most of the new configurations of connotation relations between concepts lead rather to "extinction" than to "semantic reinforcement" (please do not demand a detailed description how we imagine this; out of necessity we must use intuitive and approximate formulations and analogies; a detailed logical analysis of any system would lead to its disintegration). Thus, the formation of new concepts depends largely on the already existing structure of the conceptual network. Let us refer the above said to the question of whether mathematics is discovered or invented. A new mathematical entity appears as a new concept, and thus is “invented". However, as we have just stated, whether a new concept emerges and in what form, it is largely (if not exclusively) determined by the 
structure of the already existing conceptual network: the concept is potentially contained in it. In this sense, a new mathematical entity is "discovered" as an opportunity that has existed in the structure of the network before. The "discovery" of the existence of this opportunity leads automatically to its seizing and implementation, that is, to an "invention" of a mathematical entity. Thus, we see that the concepts of "discovery" and "invention" describe exactly the same phenomenon here. These concepts can therefore be used interchangeably in relation to mathematics, and the impression of their different meaning comes from the memory of their origin (that is, of the original meaning of the names designating them in everyday language). The above described phenomenon is very common, and is similar to making the truth or even sense out of sentences dependent on the level of hierarchy at which they are uttered, and thus — on their semantic context.

Now let us deal with a phenomenon that we will call hierarchical circular thinking. A certain introduction to its discussion was provided by the earlier discussion about the truth of some statements (for example, about the distinction between a concept and its designatum) at different levels of the hierarchy of the conceptual network. We came to the conclusion about "equality" of levels. We would now like to expand and specify this idea. We will start from the third level, that is, the everyday conceptual map. Here, all concepts are obvious and self-explanatory. All entities, both individual and general (categories, ideas), are vested with some kind of "existence". All meanings are to a large degree autonomous. Of course, at the third level, there is diversification of concepts, but there is no hierarchization of them. At this level, it is easy to determine in an "absolute" way, what is true, and what is false (or at least a potential predication of truth is possible-for example, having suitable knowledge, we can answer the question: "Is this rose red?"). When we move from the third to the second level, we encounter science, logic, and philosophy along the way. These conceptual maps are characterized by hierarchization and categorialization of concepts and entities. Some of them are granted the privilege of a "real" existence, while others (for example, often general entities) are denied such existence. At this level, there is also the problem of realism and nominalism. Truth, to some extent "absolute", can be predicated only in relation to some sentences. There are questions which we do not know how to answer; some questions are meaningless. We do not have a clear understanding which statements make sense in philosophy, and science even forbids posing certain questions. The area between the third and second level is the place of the birth of logic and of its more or less legitimate operation. But even here, it is not a universal tool. The second level (the conceptual network) is a place of a renewed flattening of the hierarchy of entities to unity. All concepts are equal here. At this level, the possibility of predicating truth in an "absolute" way is reduced to almost zero.

This leads directly to the first level, where the concept of the absolute truth becomes completely meaningless, and where, if we want to make only statements of universal validity, we should be silent. But, if the absolute truth "does not exist", relative truth is the only one we have. And as we do not have the right to demand from it any absoluteness, as this is nonsense, then the "relative" truth is as legitimate as it is possible. Here, the circle is closed and we return to the third level, where we can reasonably predicate the truth of anything. The difference lies only in the fact that the concept of absolute truth, which turned out to be empty, was replaced by the concept of "the only available" truth. However, in light of the above discussion, these concepts become practically indistinguishable at the third level. Philosophy oscillates between the first and the third level which in fact turn out to be one and the same! Thus, we have a closed loop in the hierarchy of levels. Furthermore, we could tighten this loop to a point, indicating equality of levels and the fact that they are different manifestations of our "world", which means, the totality of phenomena accessible to us. This conclusion seems to be trivial, although it is important how we came to it. Generally, in these discussions the way of drawing a conclusion is often more important than the conclusion itself, expressed in a single sentence. We have already spoken about it a couple of times.

The hierarchical circular thinking can also be used on a smaller scale. Here, we will use an example taken from moral philosophy. An immoral act, harmful to others, is obviously morally reprehensible. But if a harmful act is performed unwittingly, that is, without any intention of doing it, and not due to recklessness (for example, if we press the button to call the lift and we detonate a bomb attached to it by some terrorists, and thus we kill dozens of people), then it cannot be treated as a violation of moral standards. People are differently predestined for morality. Some are born or are socially conditioned as individuals deprived of any moral standards, and only fear of punishment can stop them from committing crimes, whereas others especially delight in doing good, this is the greatest joy for them. In this case, moral evaluation also seems questionable, as how can we praise or rebuke somebody for something that is beyond their control, innate or acquired without any possibility of choice? We say that everyone using their free will can do good, overcoming some deep temptation to commit unethical 
acts (for these acts themselves or for personal gain). But again, everyone in his or her mind finds some ready (innate or socially shaped) predisposition to overcome immoral temptations. We can say that everyone is able to shape himself or herself, but again, the predispositions to do this shaping are what they are, and so on. Thus, we return to the situation from the beginning of the circle of hierarchy, when we perform an act which is morally wrong, but independent from us. In this perspective, a premeditated killer bears the same blame as a person who is putting white poison into someone's tea, having taken it out of the box with the label "sugar". In this view, a tragic mistake turns out to be as much independent from "free will", as an act of an individual who has no predispositions to fight his or her "bad character", but is capable of recognizing that he or she is committing a criminal act. Where does the above rather cursory analysis lead? First, to desemantization of the concept of "free will"- to making an empty name out of it. Second, to a denial of applicability of any system of moral values to the human mind. But what can we do if the concepts of "free will" and "morality" are strongly established in our conceptual network, and they are intuitively obvious and "real"? A solution to this apparent contradiction is the fact that the analysed concepts are semantically empty only in the absolutist reference. However, as we have repeatedly emphasized, that reference itself is meaningless and non-legitimate. "Free will" and "morality" exist at the third level, they do not exist between the third and second level (from the point of view of science, logic and analytical philosophy), whereas at the second level the question about their existence (or non-existence) loses its meaning in an absolute way. Here, their existence is equally legitimate as the existence of a roadside poplar tree or mythological Centaurus.

To sum up, the above analysis leads to a denial of the universality of logic and to limiting its (already partial) legitimacy to the relatively narrow space between the third and second level. As we mentally "feel" the above-mentioned concepts, it means that they exist in our everyday conceptual network. Thus, there are other ways of making claims legitimate than logical analysis. Basically, it is obvious, as in a connotational system none of the reference points can aspire to universalism. In the absolute reference, all the phenomena accessible to us appear to be equally "real", both those most tangible (particular material entities) and those extremely abstract and general. There is no qualitative difference between them. Distinguishing between categories of phenomena is a result of the human mind's inclination to classify things. The absolute reference causes a flattening of the hierarchy of levels to categorial unity.

Thus, in philosophy, there is unfortunately no absolute and indisputable certainty which we can "rely on", which would make an unshakable basis and serve as our starting point or destination of our reasoning. It is impossible to come to any final conclusion, or to find an absolute base for further discussion. The situation of a philosopher closely resembles that of Baron Munchausen, who allegedly pulled himself out of a swamp by his own hair. Philosophical reflection must go in circles through subsequent levels, or settle for silence.

\section{Epilogue}

Where does all of the above said lead to? It is difficult to give a simple and unambiguous answer to this question. Instead, let us just say that we, and likewise the world for that matter, are given to ourselves as a network of concepts that have only relative meaning. Philosophy, demanding the "absolute truth" about the world, eventually questions the very fact of its existence. It is impossible to reconcile the world with the absolute truth. Therefore, it seems more reasonable to give up the latter and remain with the world as we see it in its immanence, even if we will never know what it is like "in reality".

For further reading see related articles (Korzeniewski, 2010; Korzeniewski, 2013a; Korzeniewski, 2013b).

\section{References}

Barrow, J. D. (1992). Pi in the Sky. Oxford: Oxford University Press.

Hawking, S. W. (1988). A Brief History of Time: From the Big Bang to Black Holes. New York: Bantam Books.

Hume, D. (2007). A Treatise of Human Nature. Oxford: Clarendon Press. http://dx.doi.org/10.1522/25022634

Kant, I. (1999). Critique of Pure Reason. Cambridge: Cambridge University Press.

Korzeniewski, B. (2010). From Neurons to Self-Consciousness. How the Brain Generates the Mind. Amherst: Prometheus Books.

Korzeniewski, B. (2013a). Formal Similarities between Cybernetic Definition of Life and Cybernetic Model of Self-Consciousness: Universal Definition/Model of Individual. Open Journal of Philosophy, 3, 314-328.

http://dx.doi.org/10.4236/ojpp.2013.32049 
Korzeniewski, B. (2013b). Magic of Language. Open Journal of Philosophy, 3, 455-465.

http://dx.doi.org/10.4236/ojpp.2013.34067

Mach, E. (1976). Knowledge and Error. Dordrecht: Reidel. http://dx.doi.org/10.1007/978-94-010-1428-1

Penrose, R. (1990). The Emperor's New Mind. Concerning Computers, Minds and the Laws of Physics. London: Vintage.

Piaget, J. (1955). The Language and Thought of the Child. New York: Meridian Books.

Russell, B. (1905). On Denoting. Mind, 14, 479-493. http://dx.doi.org/10.1093/mind/XIV.4.479

Russell, B. (1908). Mathematical Logic as Based on the Theory of Types. American Journal of Mathematics, 30, $222-262$. http://dx.doi.org/10.2307/2369948

Russell, B. (1975). My Philosophical Development. New York: Simon and Schuster.

Whitehead, A.N. \& Russell, B. (1910-1913). Principia Mathematica. Cambridge: Cambridge University Press.

Wittgenstein, L. (1961). Tractatus Logico-Philosophicus. New York: Humanities Press.

Wittgenstein, L. (1999). Philosophical Investigations. Upper Saddle River, NJ: Prentice Hall. 
Scientific Research Publishing (SCIRP) is one of the largest Open Access journal publishers. It is currently publishing more than 200 open access, online, peer-reviewed journals covering a wide range of academic disciplines. SCIRP serves the worldwide academic communities and contributes to the progress and application of science with its publication.

Other selected journals from SCIRP are listed as below. Submit your manuscript to us via either submit@scirp.org or Online Submission Portal.
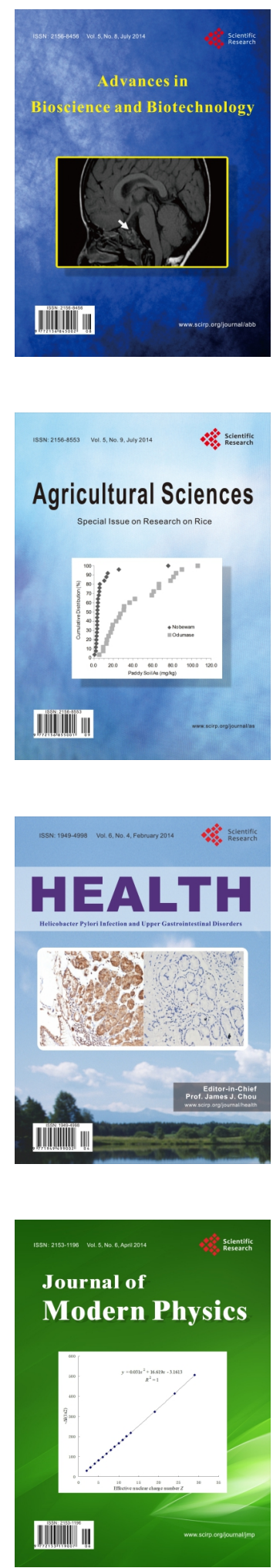
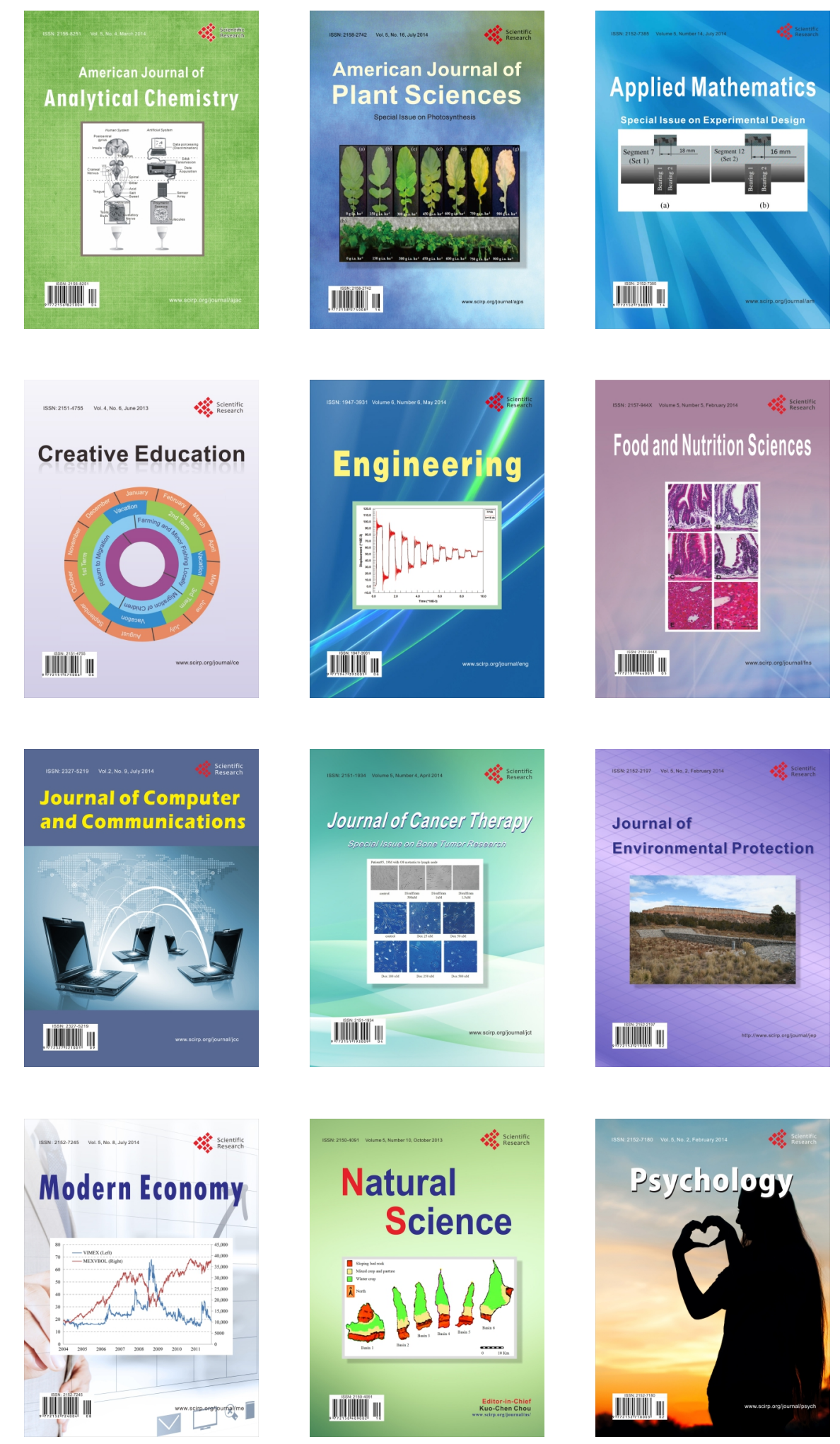